\title{
Orally Fed Recombinant Lactococcus lactis Displaying Surface Anti-Fimbrial Nanobodies Protects Piglets against Escherichia coli Causing Post-Weaning Diarrhea
}

\author{
Emmanuel Okello ${ }^{1,2,3,4} \mathbb{D}$, Kristof Moonens ${ }^{1,2,+}$, Joseph Erume ${ }^{4}$ and Henri De Greve ${ }^{1,2, *}$ \\ 1 VIB-VUB Center for Structural Biology, Pleinlaan 2, 1050 Brussels, Belgium; eokello@ucdavis.edu (E.O.); \\ kristof.moonens@gmail.com (K.M.) \\ 2 Structural Biology Brussels, Vrije Universiteit Brussel, Pleinlaan 2, 1050 Brussels, Belgium \\ 3 Department of Population Health and Reproduction, School of Veterinary Medicine, University of California \\ Davis, 1 Garrod Dr., Davis, CA 95616, USA \\ 4 College of Veterinary Medicine, Animal Resources and Bio-security, Makerere University, P.O. Box 7062 \\ Kampala, Uganda; jerume@covab.mak.ac.ug \\ * Correspondence: Henri.De.Greve@vub.be; Tel.: +32-2-6291844 \\ † Present address: Ablynx, Technologiepark 21, 9052 Ghent/Zwijnaarde, Belgium.
}

\section{check for}

updates

Citation: Okello, E.; Moonens, K.; Erume, J.; De Greve, H. Orally Fed Recombinant Lactococcus lactis Displaying Surface Anti-Fimbrial Nanobodies Protects Piglets against Escherichia coli Causing Post-Weaning Diarrhea. Agriculture 2021, 11, 186. https://doi.org/10.3390/agriculture 11030186

Academic Editors: Nicole Kemper and Grzegorz Woźniakowski

Received: 28 January 2021

Accepted: 19 February 2021

Published: 24 February 2021

Publisher's Note: MDPI stays neutral with regard to jurisdictional claims in published maps and institutional affiliations.

Copyright: (c) 2021 by the authors. Licensee MDPI, Basel, Switzerland. This article is an open access article distributed under the terms and conditions of the Creative Commons Attribution (CC BY) license (https:// creativecommons.org/licenses/by/ $4.0 /)$.
Abstract: Post-weaning diarrhea (PWD) and edema disease (ED), caused by enterotoxigenic and Shiga toxin producing Escherichia coli (ETEC and STEC) strains, are important diseases of newly weaned piglets worldwide. The objective of this study is to develop a passive immunization strategy to protect piglets against PWD and ED using recombinant Lactococcus lactis added to piglet diet at weaning. The Variable Heavy chain domains of Heavy chain antibodies (VHHs) or Nanobodies (Nbs), directed against the fimbrial adhesins FaeG (F4 fimbriae) and FedF (F18 fimbriae) of E. coli were cloned and expressed on the surface of L. lactis. In vitro, the recombinant L. lactis strains agglutinated and inhibited adhesion of cognate F4 or F18 fimbriae expressing E. coli to pig villous preparation. In vivo, the anti-F4 L. lactis protected weaned piglets against a challenge with an F4-positive ETEC strain. Piglets supplemented with oral anti-F4 L. lactis showed reduced fecal E. coli shedding. We concluded that the surface expressed Nanobodies on L. lactis neutralized the adhesins of targeted E. coli and abrogated gut colonization, the first step in disease pathogenesis. As a proof of concept, we demonstrated the potential of passive immunization with recombinant $L$. lactis as a viable alternative to antibiotic prophylaxis in preventing piglet-post-weaning diarrhea.

Keywords: post-weaning diarrhea; edema diseases; ETEC; STEC; VHH; Nanobodies; passive immunization; Lactococcus

\section{Introduction}

Post-weaning diarrhea (PWD) and edema disease (ED) caused by enterotoxigenic and Shiga toxin producing Escherichia coli (ETEC and STEC) are a major cause of economic losses to pig farmers worldwide [1]. The losses result from mortality, morbidity, and the cost of treatment. The key virulence factors of these pathogenic strains are F4 and/or F18 fimbriae expressed in various combinations with heat labile toxin (LT), heat stable toxin (STa \& STb) and Shiga toxin (Stx2e) [2-7]. Fimbriae are multi-subunit proteinaceous surface appendages that bind to specific receptors on enterocytes, enabling the bacteria to colonize the gut epithelium, the first step in disease pathogenesis [3]. Following colonization, the strains express toxins that induce secretory diarrhea or edema disease by interfering with water and electrolyte transport across epithelial cells, or toxic endothelial cell apoptosis, respectively [8-10].

Fimbriae are highly immunogenic and stimulate both serum and mucosal immune responses [11]. The peak antibody levels occur at 1 week (F4-specific IgA) and 3 weeks (F18-specific IgA) post infection respectively [12]. This immune response is dependent on 
receptor recognition of the antigen, since the receptor negative piglets tolerate fimbriae as normal food antigens [13]. Vaccination of sows with fimbriae or fimbrial subunits is the current strategy for protecting neonatal piglets from ETEC infections through the induced lactogenic immunity [14]. However, active immunization inducing an effective intestinal mucosal immunity is required to protect post-weaning piglets following withdrawal of milk antibodies [15]. The experimental immunization of piglets orally with purified F4 fimbriae or the adhesive subunit (FaeG) induced protective F4-specific mucosal immune response in piglets $[16,17]$. Similarly, oral immunization with purified F18-fimbriae induced specific immune responses to the major subunit FedA, but the immune response against the FedF adhesin was weak and non-protective against F18+ E. coli challenge [18].

During the suckling period, antibodies in milk neutralizes oral vaccines and prevent the development of protective mucosal immunity. Since the diseases occur immediately after weaning, there is no sufficient time for the piglets to mount an immune response, and thus precludes the use of active immunization to prevent post-weaning diarrhea. Attempts to circumvent this problem using an encapsulated or enteric coated subunit and live vaccines resulted in improved immune response in mice models [19] and partial protection of challenged piglets $[15,20]$.

Oral immunization of suckling piglets with E. coli or fimbriae, microencapsulated using spray dried poly (lactide-co-glycolide) microspheres, did not induce significant antibody levels 19 days after the booster dose, and the piglets were not protected from challenge post-weaning [21]. Experiments in mice showed that the microencapsulation of F4 and F18 fimbriae with thiolated eudragit microspheres for oral immunization resulted in increased antigen-specific IgA and IgG in serum and proliferation of immune cells in the spleen, lamina propria and the Peyer's patches [19]. Although the results were promising, the results were not validated in suckling piglets in the presence of milk antibodies and other inhibitory factors.

Passive immunization protects against gut colonization following withdrawal of milk antibodies at weaning, allowing the piglets to adapt to stressful changes during this period. Subsequent challenge with pathogenic strains would be better tolerated, since older piglets mount better immune responses and the fimbrial receptors diminish with age [22]. Passive immunization by the in-feed addition of serum-dried plasma antibodies or egg yolk antibodies from immunized chicken during the first two to three weeks after weaning was shown to prevent PWD $[23,24]$. However, the use of animal products in feed for farm animals is limited by the cost of production and regulatory restrictions, due to public health concerns [25]. Cost effective passive immunization can be achieved using recombinant antibodies expressed cheaply in microbial systems. Lactococcus is the microbial system of choice, due to its GRAS (Generally Regarded As Safe) status. Recent research studies have demonstrated the potential for use of engineered Lactococcus strains for passive immunization by secreting the antigens [26] or displaying subunit antigens and antibody fragments on the bacterial surface using anchor proteins [27-29].

The variable domains derived from llama Heavy chain antibodies (HCAb), also known as Nanobodies (Nbs), fused to Fc domains are suitable proteins for passive immunization, due to the ease of expression and scale up for bulk production in plant and yeast systems [30]. The HCAb consists only of the two heavy chains and lacks the two light chains found in conventional antibodies [31]. Nanobodies are derived from the variable domain of $\mathrm{HCAb}$ and can bind antigens despite having only $\mathrm{VH}$ domains. They represent a large diversity in their structural repertoire of antigen-binding sites and antigen-binding characteristics [32]. Nanobodies are small proteins (15 kDa) that are very soluble and very stable allowing for ease of expression in various heterologous systems. Nanobodies have recently gained wide application in animal production for research, diagnostics, therapy, and immunization. Studies reporting on diagnostic applications include the development of a specific nanobody for rapid and selective determination of Salmonella enteritidis in milk and a nanobody-based lateral flow assay to detect active Trypanosoma congolense infections in cattle $[33,34]$. Similarly, several studies have reported on the use of Nanobodies 
for passive immunization, through systemic or oral administration, to protect animals against viral and bacterial infections such as foot and mouth disease, gastroenteritis, and Clostridium difficile [35-39].

The goal of this study is to develop a passive immunization strategy against postweaning diarrhea in piglets using neutralizing Nanobodies, directed against fimbrial adhesins FaeG and FedF. All the Nanobodies used in this study were developed in our laboratory, with known structures and inhibitory properties [40,41]. Related studies had already demonstrated the neutralizing and protective effect of anti-FaeG $\mathrm{Nb}$ expressed in Arabidopsis thaliana and yeast cells, when added to feeds during weaning period $[35,37]$. These Nbs were expressed on Lactococcus lactis by surface display using the anchor domain of protein A of Staphylococcus aureus [42]. When added in feeds, the covalently linked Nbs on the L. lactis surface specifically recognized and neutralized the fimbrial adhesins on targeted ETEC and STEC strains and prevented intestinal colonization, the first step in disease pathogenesis.

\section{Materials and Methods}

The fusion genes of different $\mathrm{Nbs}$ and protein $\mathrm{A}$ (ProtA) sequences from S. aureus were inserted into pTRKH3 shuttle vector [43] adapted in-house to the Gateway cloning system (Thermo Fisher Scientific) and transformed into the expression strain L. lactis MG1363 [44]. pTRKH3 is a shuttle cloning vector for E. coli and Gram-positive bacteria with a high copy number (45-85) in streptococcal and lactococcal hosts. The vector contains tetracycline and erythromycin genes; tetracycline resistance is only expressed in E. coli, while erythromycin resistance is expressed both in E. coli and Gram-positive bacteria.

\subsection{Construction of Nb-ProtA Recombinant Fusion Genes}

The $\mathrm{Nb}$ coding sequences were amplified from plasmids containing the inserts. These Nbs were generated in our laboratory and are well characterized [40,41]. The anchor domain of ProtA were amplified from total genomic DNA of S. aureus NTC8325 [45] using primer pair $\operatorname{coc} 3$ and $\operatorname{coc} 5$. The $\mathrm{Nb}$ coding sequences were amplified using primers $\operatorname{coc} 2$ and coc4 from miniprep DNA. The primers $\operatorname{coc} 4$ and $\operatorname{coc} 3$ were constructed with the respective $3^{\prime}$ and $5^{\prime}$ ends extension complementary to a segment of the $\mathrm{Nb}$ and ProtA sequences, and $\operatorname{coc} 2$ had $5^{\prime}$ extension of Usp45 signal sequence. $\mathrm{Nb}$ and ProtA PCR fragments were joined in an overlap PCR reaction using primers pair coc1 and coc5. Primers $\operatorname{coc} 1$ and $\operatorname{coc} 5$ had, respectively, a $5^{\prime}$ and $3^{\prime}$ extension of attB sequence of the gateway system. The primer sequences and the PCR conditions are provided in Appendix A. The cloning scheme is shown in Figure 1.

The attB flanked $\mathrm{Nb}$-ProtA fusion genes were cloned into the Gateway vector pDONR221 (Thermo Fisher Scientific, Merelbeke, Belgium) in a BP reaction according to the Gateway Technology manual (Thermo Fisher Scientific, Merelbeke, Belgium, Version E, Catalog nos.12535-019 and 12535-027). Briefly, the following reaction components were set up in $1.5 \mathrm{~mL}$ microcentrifuge tubes for each sample: $1.0 \mu \mathrm{L}$ AttB-PCR product $(66 \mathrm{ng} / \mu \mathrm{L})$, $1.0 \mu \mathrm{L} \mathrm{pDONR}^{\mathrm{TM}}$ vector $(150 \mathrm{ng} / \mu \mathrm{L})$, and $8.0 \mu \mathrm{L}$ TE Buffer $\mathrm{pH}$ 8.0. The product of BP reaction $(5 \mu \mathrm{L})$ was used to transform $\mathrm{CaCl}_{2}$-competent $\mathrm{DH} 5 \alpha$ cells [46] and transformed colonies selected on LB agar plates with kanamycin. Presumptive pENTR clones were screened by colony PCR using SeqLA1 (5'CTCTGCCGTTAACGCTAGCATGGAT3 ${ }^{\prime}$ ) and SeqLB (5'GTAACATCAGAGATTTTGAGACAC3') primers and positive clones confirmed by sequencing (Gene Sequencing Facility, University of Antwerp, Antwerp, Belgium). Sequence analysis of the pENTR clones showed variability in the ProtA sequence length. To standardize ProtA lengths, one short and long $\mathrm{Nb}$-Prot A were selected and exchanged with each of the $\mathrm{Nb}$-ProtA fragments of all the $\mathrm{pENTR}$ clones by Gibson assembly reactions (Appendix A). 


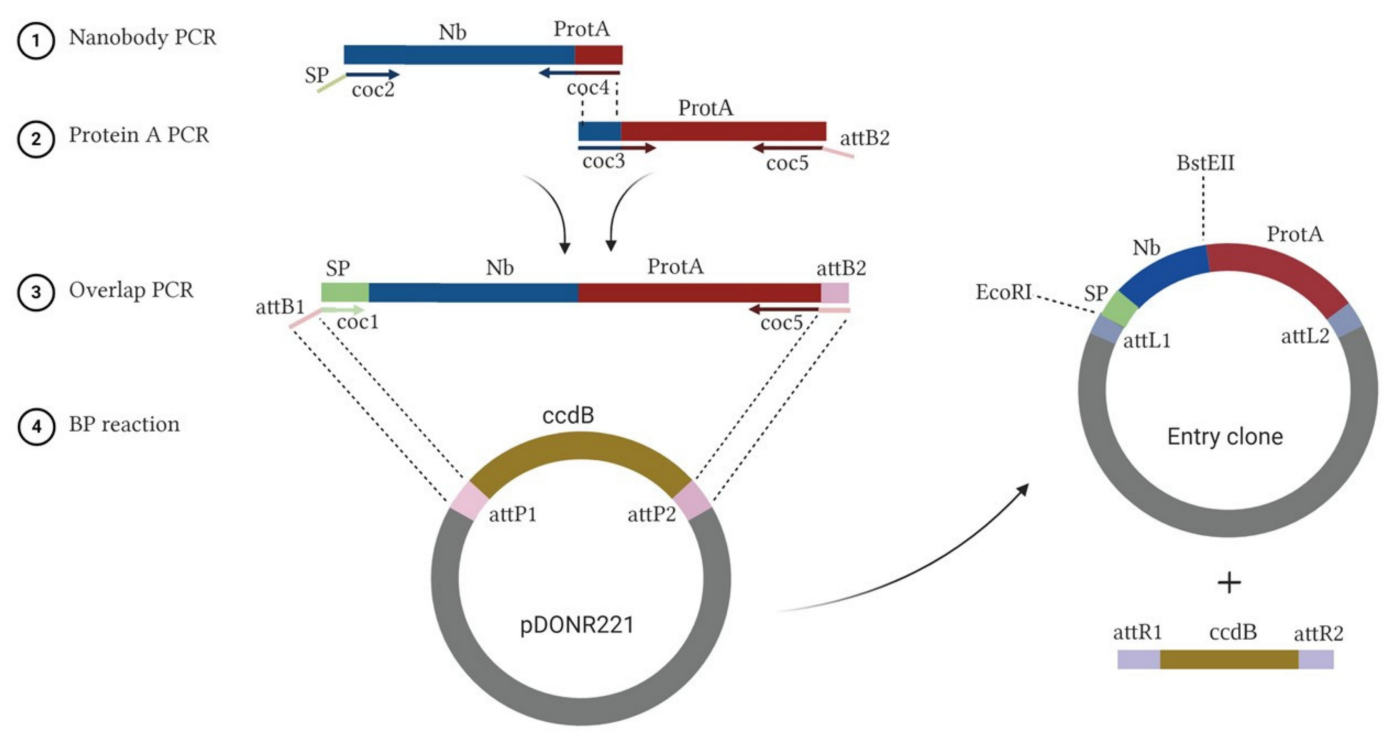

Figure 1. Cloning of Nanobodies (Nb)-protein A (ProtA) fusion gene for surface display in L. lactis. The signal peptide (SP), the $\mathrm{Nb}$ genes and the anchor domain of ProtA were amplified in separate PCR reactions and the gene fragments were fused in an overlap PCR reaction introducing the attB sites of the Gateway system at both ends. Created in BioRender.

\subsection{LR Reaction between $p D O N R 221$ Containing Nb-ProtA Insert and Gateway Adapted pTRKH3 Vector}

The Nb-ProtA constructs in pDONR221 vector (pENTR) were transferred using a LR reaction into the Gateway-adapted pTRKH3 expression vector pHD669 carrying the Lactococcus clpC promoter upstream the Gateway cassette. The detailed procedure for adapting vector pTRKH3 to the Gateway system is provided in Appendix A. The LR reaction protocol was set up according to the Gateway Technology manual (Thermo Fisher Scientific,, Merelbeke, Belgium, Version E, Cat. $\neq 12535-019)$. Briefly, the following components of the reaction solution was added to 1.5 microcentrifuge tube: $6 \mu \mathrm{L}$ of entry clone (Nb-ProtA in pDONR221), $2 \mu \mathrm{L}$ of Gateway-adapted pHD669, $4 \mu \mathrm{L}$ 5X LR Clonase reaction buffer, $4 \mu \mathrm{L}$ TE buffer, $\mathrm{pH} 8$, and $4 \mu \mathrm{L}$ LR Clonase enzyme. The reaction solution was mixed well and incubated at $25^{\circ} \mathrm{C}$ for $1 \mathrm{~h}$, after which Proteinase $\mathrm{K}$ was added and incubated for $10 \mathrm{~min}$ at $37^{\circ} \mathrm{C}$ to stop the reaction. Ten $\mu \mathrm{L}$ of the LR reaction product were transformed into $\mathrm{DH} 5 \alpha \mathrm{CaCl}_{2}$-competent cells and selected on LB media supplemented with tetracycline. Miniprep DNA prepared from transformants were submitted for sequencing (Gene Sequencing Facility, University of Antwerp, Antwerp, Belgium) using primer pair pTRKH8 (5'CTAATAAAGCCGTAAGGAGACGGGTTCA3') and pTRKH9 (5'CCCATCCTAACGGCCACG CATATG3'). Miniprep DNA prepared from expression clones in DH5 $\alpha$ were used to transform electro-competent L. lactis MG1363 [44]. Electroporation was conducted at $2.5 \mathrm{kV}, 200 \Omega$, and $25 \mu \mathrm{F}$. Positive clones were selected on M17 media supplemented with $0.5 \%$ glucose and $10 \mu \mathrm{g} / \mathrm{mL}$ erythromycin and confirmed by colony PCR.

\subsection{Validation of $\mathrm{Nb}$ Surface Display on L. lactis}

\subsubsection{Slide Agglutination Assay}

Recombinant L. lactis strains were grown on M17 broth (Oxoid ${ }^{\circledR}$, Thermo Fisher Scientific, Merelbeke, Belgium) supplemented with glucose $(0.5 \%)$ and erythromycin $(5 \mu \mathrm{g} /) \mathrm{mL}$ for $48 \mathrm{~h}$ at $30^{\circ} \mathrm{C}$. Cells were pelleted at $2000 \mathrm{rpm}$, washed once with 10 $\mathrm{mL}$ phosphate buffered saline (PBS), and re-suspended in PBS. Cell concentration was estimated by $\mathrm{OD}$ measurement and was adjusted to $\mathrm{OD}_{600}=3$. High cell concentrations enabled the visualization and scoring of the agglutination effect. In the agglutination test, $15 \mu \mathrm{L}$ of the prepared L. lactis was added to an equal volume of $E$. coli cell suspension on an 
agglutination plate and mixed by rocking for 1-3 min. Positive agglutination was observed visually against a light source and scored objectively on the basis of time of appearance and intensity. The scores ranged from 1-5 ( 5 = rapid uniform agglutination within 15 sec; $4=$ rapid uniform agglutination within $30 \mathrm{sec} ; 3=$ uniform agglutination after $30 \mathrm{sec}-1$ $\min ; 2=$ limited agglutination after $1 \mathrm{~min} ; 1=$ apparent agglutination seen after $1 \mathrm{~min} ; 0=$ no agglutination after $3 \mathrm{~min}$ ).

\subsubsection{In vitro Villous Adhesion Assay}

In vitro villous adhesion assay was performed as previously described $[22,47,48]$. The cell concentration was adjusted to $10^{10}$ cells/ $\mathrm{mL}$ for L. lactis and $10^{8}$ cells/ $\mathrm{mL}$ for E. coli using OD estimates. The intestinal villi were prepared from the mid-jejunum of four-weekold piglets (provided by Prof. Dr. Eric Cox, Ghent University). The villi from each piglet were tested for the presence of the F4 (three variants F4ab, F4ac and F4ad) and the F18 receptors. Only villi from piglets positive for the F4 and F18 receptors were used in the adhesion assay. The intestinal sections were sliced, rinsed in PBS, and scraped into KrebsHenseleit buffer containing $1 \%$ formaldehyde and stored at $4{ }^{\circ} \mathrm{C}$. Before use, the thick villi suspension was washed twice with Krebs-Henseleit buffer without formaldehyde and re-suspended in PBS. For each assay, a mixture of $100 \mu \mathrm{L}$ F4 or F18 E. coli with $100 \mu \mathrm{L}$ of respective anti-F4 or anti-F18 L. lactis was incubated for $1 \mathrm{~h}$ at RT on atating plate (50 rpm), after which $50 \mu \mathrm{L}$ prepared villous were added to the cell mixture and topped to $500 \mu \mathrm{L}$ with PBS, and incubated for additional $1 \mathrm{~h}$ at RT. For the controls, anti-F4 and anti-F18 L. lactis were pre-incubated with $50 \mu \mathrm{g}$ FaeG and FedF, respectively, for $15 \mathrm{~min}$ at RT on a slow rotating plate $(50 \mathrm{rpm})$ before adding the $E$. coli strains and the subsequent steps were followed as above. To detect adherence, $12 \mu \mathrm{L}$ of the prepared sample were observed under light microscopy and the number of E. coli cells adhering to $250 \mu \mathrm{m}$ villous length was counted at $\times 100$ magnification in oil immersion. Adhesion of $<5,5-30$, and $>30$ E. coli cells per $250 \mu \mathrm{m}$ brush border length was considered as negative, weak, and strongly positive, respectively. The mean difference in the number of E. coli cells adhered to $250 \mu \mathrm{m}$ villous length between the test (adhesion inhibition assay) and controls (reversal of adhesion inhibition) were compared using paired t-tests.

\subsubsection{Fluorescence Microscopy of Recombinant L. lactis}

Surface display of $\mathrm{Nbs}$ on L. lactis was visualized by fluorescence microscopy using specific $\mathrm{Nb}$ antigen (FaeG or FedF) labeled with FITC (Flourescien-5-isothiocyanate). FaeG and FedF were conjugated with FITC according to the manufacturer's manual (Molecular Probes by Invitrogen; Cat.\# F1906, Lot. 513684). Briefly, purified FaeG $(8.1 \mathrm{mg} /) \mathrm{mL}$ and FedF (5.7 mg/)mL subunits in $20 \mathrm{mM}$ Tris buffer were exchanged into $0.1 \mathrm{M}$ sodium bicarbonate buffer (pH 8.5) using Zeba ${ }^{\mathrm{TM}}$ Spin Desalting Columns, 7K MWCO (Thermo Fisher Scientific, Lot\# 203162, Merelbeke, Belgium) and concentrations adjusted to $5 \mathrm{mg} / \mathrm{mL}$ FITC dye were dissolved in DMSO to a final concentration of $10 \mathrm{mg} / \mathrm{mL}$, and $10 \mu \mathrm{L}$ of the solution were added slowly with continuous vortexing to $200 \mu \mathrm{L}$ FaeG and FedF preparations. The reaction mix was incubated at $\mathrm{RT}$ for $1 \mathrm{~h}$ on a slow rotating plate. Conjugated protein was purified using illustra ${ }^{\mathrm{TM}} \mathrm{NAP}^{\mathrm{TM}}-5$ columns (GE Healthcare, Diegem, Belgium) to remove free dye. The L. lactis cells were prepared as described for the in vitro adhesion assay and resuspended in PBS to a concentration of $1 \times 10^{8}$ cells/.mL The cells were blocked with 3\% BSA for $20 \mathrm{~min}$, labeled antigens added to final concentration of $2 \mu \mathrm{g} / \mathrm{mL}$ incubated for additional $20 \mathrm{~min}$, washed $2 \times$ and resuspended in PBS. All washing steps were performed in PBS and centrifugation at $2000 \mathrm{rpm}$ for $7 \mathrm{~min}$ at $4{ }^{\circ} \mathrm{C}$. Fifteen microliters of labeled cell suspension was applied to a microscope slide and viewed under inverted fluorescence microscope (Nikon Eclipse TE2000-U, Groot-Bijgaarden, Belgium), at 100× magnification, and image captured using attached camera. 


\subsection{In vivo Piglet Challenge Experiments}

A clinical trial was conducted to evaluate the protectiveness of the recombinant anti$\mathrm{F} 4$ L. lactis in weaned piglets challenged with an $\mathrm{F}^{+}$E. coli. Only anti-F4 L. lactis strains were tested in the challenge experiment based on the known challenge model for the F4-fimbriated ETEC strains [37].

\subsubsection{Animal Preparation}

The research protocol was approved by the Uganda National Council for Science and Technology (UNCST) on 16 April 2013; Approval \#HS 1361. Twenty piglets from 3 sows were weaned at 4 weeks and transported to the animal facility at the College of Veterinary Medicine, Makerere University. The piglets were drawn from 3 different litters and comprised of both males and females. The piglets were tagged and divided into 3 groups of 5, 5 and 10 animals (Table 1). The different groups were housed in disinfected, separate pens within the same housing unit. The different pens were separated by partial solid walls (1.25 m height) that precluded physical contact between piglet groups. Group 3 was split into 2 pens of 5 piglets each. To clear possible gut ETEC E. coli infection present at weaning, the piglets were treated with Enrofloxacin oral drench $(5 \mathrm{mg} / \mathrm{kg})$ for 3 consecutive days beginning 2 days prior to weaning. This was necessary because there were no known F4 or F18 E. coli negative farms. Enrofloxacin was chosen because all pathogenic E. coli strains isolated from pig farms in this region had tested susceptible to fluoroquinolones [49]. Piglets from the same litter were distributed equally in the different groups to account for cohort effect.

Table 1. Piglet groups and treatment.

\begin{tabular}{cccc}
\hline Group & Number & Treatment & L. lactis Dose \\
\hline 1 (Negative control) & 5 & No F4 ETEC given & No L. lactis added \\
2 (Positive control) & 5 & F4 ETEC + MG1363 L. lactis & $10^{10}$ cells $/ 100 \mathrm{~g}$ of feed \\
3 (Test) & 10 & F4 ETEC + Anti-F4 L. lactis & $10^{10}$ cells $/ 100 \mathrm{~g}$ of feed \\
\hline
\end{tabular}

\subsubsection{Feeding and Challenge}

All piglets were fed commercial weaner's meal supplied locally at a rate of 400 $\mathrm{g} /$ day divided in 2 daily rations (fed in the morning and evening), as per the supplier's recommendation. Weaners' meal comprised of maize bran base, protein and mineral supplements. For the treated group, the anti-F4 L. lactis suspension in $10 \mathrm{~mL}$ of PBS $\left(10^{10}\right.$ cells $/ 100 \mathrm{~g}$ of feed) was added and mixed in the feed with additional water to a uniform soft consistency. In the control groups, only PBS was added to the feed and additional water added to soft consistency as above. L. lactis strains were added to feeds starting two days before the challenge with the F4-positive strain and continued till day 18 after challenge.

Starting on day 0 (weaning day), the piglets were inoculated daily with a mix of L. lactis strains in feed at a dose of $10^{10}$ cells $/ 100 \mathrm{~g}$ of feed. Cell concentration was estimated from OD measurements. On day 2 and 3, the piglets (test and positive control groups) were challenged with $10^{9} \mathrm{~F} 4^{+}$E. coli strain (C95-72) by oral drenching. The E. coli cells were harvested from an overnight culture incubated at $37^{\circ} \mathrm{C}$. L. lactis strains were grown in M17 medium supplemented with $0.5 \%$ glucose (final concentration) at $30^{\circ} \mathrm{C}$ for $48 \mathrm{~h}$.

\subsubsection{Monitoring Response}

The piglets were weighed daily and monitored daily for any signs of diarrhea or edema disease. Fecal samples were also collected daily from each piglet for total bacterial counts. $\mathrm{F}^{+}$E. coli isolates in fecal samples were confirmed by slide agglutination test using anti-F4 L. lactis on 10 selected clones per piglet. Blood samples were taken from the jugular vein on days 0,7 , and 14 post-challenge to evaluate immune response by. On day 15 , the piglets were sacrificed and jejunal villi isolated to confirm F4 receptor status 
by in vitro adhesion assay, as previously described [22]. The Enzyme-Linked Immuno Sorbent Assay (ELISA) protocol used was previously described by the authors [49]. Briefly, we ran indirect ELISA serial dilutions of a known positive and negative serum samples, and all the test samples, to establish the optimum dilution that would allow an optimal comparison. Subsequently, we chose a single point dilution (1:10) in serum dilution buffer $(\mathrm{PBS}+0.05 \%(v / v)$ Tween $20+3 \% w / v$ BSA) and ran all the samples again in duplicate plates concurrently under identical conditions to minimize variations. The serum samples were pre-treated with kaolin before dilution. The plates (Nunc MaxiSorp, Thermo Fisher Scientific, Merelbeke, Belgium) were coated with $1.5 \mu \mathrm{g} / \mathrm{mL}$ of purified recombinant FaeGac in coating buffer (100 mM bicarbonate/carbonate buffer; $\mathrm{pH} 9.6)$ for $3 \mathrm{~h}$ at $37^{\circ} \mathrm{C}$ and blocked overnight at $4{ }^{\circ} \mathrm{C}$ with PBS $+0.2 \%(v / v)$ Tween 80 . Serum samples were applied, and plates incubated at $37^{\circ} \mathrm{C}$ for one hour. Rabbit anti-pig IgG HRP conjugated secondary antibody (1:10,000 dilution) was then added and incubated at $37^{\circ} \mathrm{C}$ for $1 \mathrm{~h}$, followed by the substrate $\left(3,3^{\prime}, 5,5^{\prime}\right.$-tetramethylbenzidine (TMB, Invitrogen) for $20 \mathrm{~min}$ at $\mathrm{RT}$, and the reaction was stopped with an equal volume of $1 \mathrm{~N} \mathrm{HCl}$. Optical density (OD) was read at $450 \mathrm{~nm}$. The plates were washed twice with PBS between each incubation step.

\subsubsection{Statistical Analysis}

Experimental data were recorded in Microsoft Excel and analyzed using Stata IC 15 (Stata Corp LLC, College Station, TX, USA) and GraphPad Prism 9.0 statistical software. Repeated measures of body weight and fecal bacterial shedding were summarized using descriptive statistic means for continuous variables. Uncertainty measures including standard errors and $95 \%$ Confidence Intervals (CI) were also reported. The analysis of variance (ANOVA) test was used to compare the differences in weight gain, fecal bacterial shedding, and immune response between the different treatment groups. Mixed effects logistic regression models were used to predict changes in weight gain, immune response, and fecal bacterial shedding; treatment, experiment day, and interaction between treatments and experiment day were the fixed factors, and individual animals were the random factors. Checks on data normality distribution showed that the measure on fecal bacteria shedding was skewed to the right and was hence transformed to decadic logarithm for the final analysis.

\section{Results}

\subsection{Cloning $\mathrm{Nb}$ for Surface-Display on L. lactis}

The current study was initiated by the design of L. lactis recombinant strains that covalently present anti-FedF or anti-FaeG (respective adhesive subunits of F18 or F4 fimbriae) Nanobodies on their cell surface. The Nanobodies were cloned in pTRKH3 shuttle vector adapted to the Gateway system (pHD669) for expression in L. lactis. The vector $\mathrm{pHD} 669$ contain the clpC promoter from L. fermentum BR11. This promoter displayed high activity in all Lactobacillus strains tested when present as a transcriptional fusion with the gus A gene [50]. The fusion gene constructs consisting of a G-positive signal peptide, the $\mathrm{Nb}$, and the protein $\mathrm{A}$ anchor domain derived from $S$. aureus sequences were expressed from this promoter (Figure 2).

We used both the long (163 amino acid residues) and short (63 amino acid residues) ProtA anchor domain to construct different recombinant L. lactis strains (Table 2) and compared the effect of the anchor length on biological activity. Complete protein sequence alignment of the fusion gene constructs for anti-FaeG and anti-FedF Nanobodies are shown in Supplementary Figure S1 and Figure S2, respectively. 

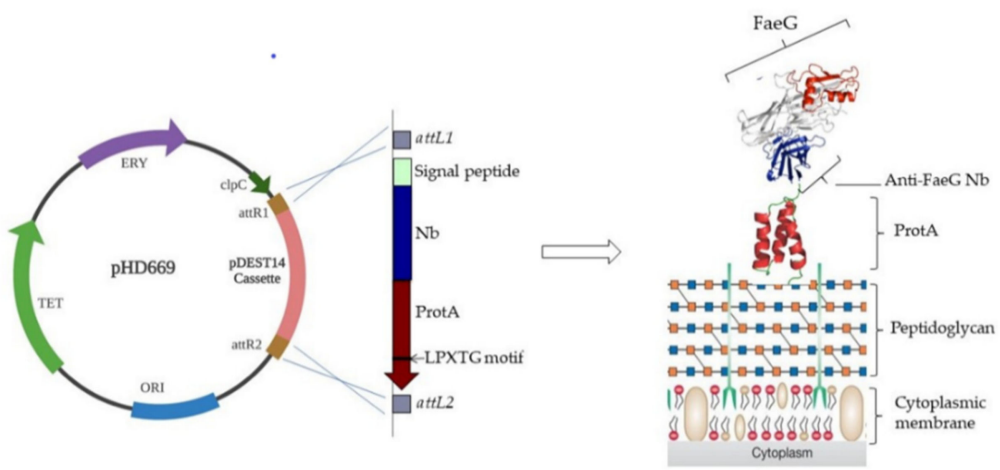

Figure 2. Cloning scheme for $\mathrm{Nb}$ surface display on L. lactis cell wall using ProtA anchor domain. Left: The pTRKH3 shuttle vector was adapted to the Gateway cloning system by inserting the pDEST14 cassette flanked by the attR1 and attR2 recombination sites, downstream of the cplC promoter sequence, in the ApaLI restriction site to form plasmid pHD669. The fusion gene comprised the UspA signal peptide sequence for Gram-positive bacteria (green), $\mathrm{Nb}$ (blue) sequence, and protein $\mathrm{A}$ anchor domain sequence (red). The C-terminus of protein A contained the LPXTG motif (X is any amino acid) that is recognized by sortase, a transaminopeptidase enzyme, that cleaves the protein A between the threonine and glycine residues of the motif and links it covalently to the pentaglycine peptide of the cell wall peptidoglycan. The pentaglycine peptide is an interbridge between the tetrapeptides that cross-link the N-acetylglucosamine (NAG) and N-acetylmuramic acid (NAM) polysaccharide chains to form the lattice-like structure of the cell wall. On the right is a schematic presentation of the anti-FaeG Nb (blue) in the complex with the FaeG subunit from F4 fimbriae (in grey is the immunoglobin like core and in red is the receptor binding domain) [41] anchored on the cell wall [51] by protein A (red) [52].

Table 2. Different recombinant L. lactis strains expressing Nanobodies against FaeG and FedF anchored on the cell surface via a short or long protein A protein linker.

\begin{tabular}{cccc}
\hline \multicolumn{2}{c}{ Anti-FaeG L. lactis Strains } & \multicolumn{2}{c}{ Anti-FedF L. lactis Strains } \\
\hline Long Anchor ProtA & Short Anchor ProtA & Long Anchor ProtA & Short Anchor ProtA \\
\hline pEXP366 (V2) & pEXP444 (V2) & pEXP362-1 (NbFedF6) & pEXP447 (NbFedF9) \\
pEXP368 (V4) & pEXP445 (V3) & pEXP364-3 (NbFedF7) & pEXP451 (NbFedF12) \\
pEXP441 (V1) & pEXP455 (V1) & pEXP459 (NbFedF9) & pEXP449 (NbFedF11) \\
pEXP457 (V4) & & pEXP463 (NbFedF12) & pEXP365 (NbFedF7) \\
& & pEXP461 (NbFedF11) & pEXP363 (NbFedF6) \\
\hline
\end{tabular}

\subsection{In vitro Assay to Validate Nanobodies Surface Display on L. lactis}

We demonstrated the expression and functional surface display of the $\mathrm{Nbs}$ on L. lactis using slide agglutination test, immunofluorescence, and flow cytometric analysis.

\subsubsection{Agglutination Assay}

To cause agglutination, the surface $\mathrm{Nbs}$ on L. lactis needs to specifically recognize FedF or FaeG subunits of F4 or F18 fimbriae present on E. coli. In our agglutination assay, overnight cultures of L. lactis and E. coli strains were harvested, washed, and re-suspended in PBS at $\mathrm{OD}_{600}$ 3. Equal volumes of L. lactis and the E. coli suspension were added into wells of agglutination plates, mixed by gentle rocking, and observed agglutination against light source. The agglutination was scored on the basis of the time of appearance and the extent of granulation. Cross-linking of several E. coli and Lactococcus cells created a mesh forming macroscopic granules. The result of the agglutination assay is shown in Figure 3. The rest of the L. lactis constructs was tested for agglutination with different E. coli strains, and the results are summarized in Supplementary Tables S1 and S2. 

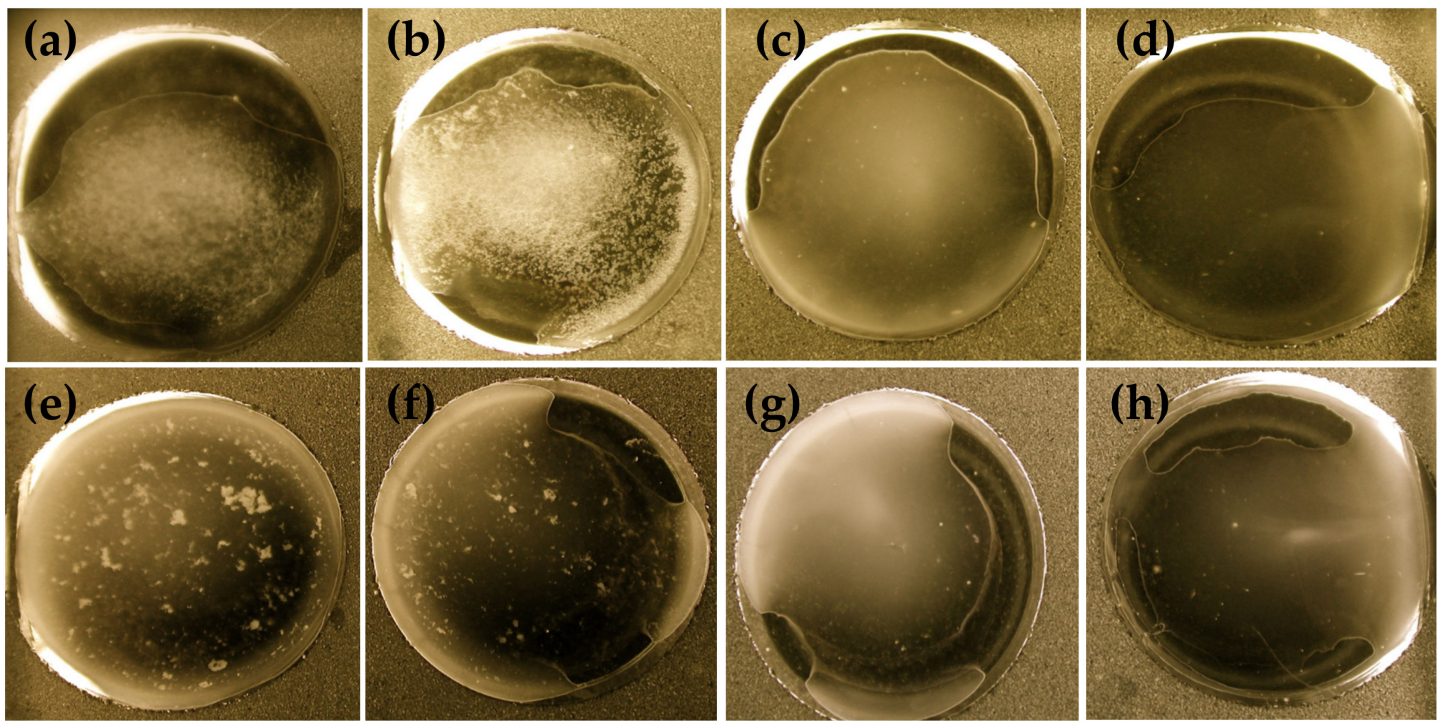

Figure 3. Agglutination of $\mathrm{F}^{+}$and $\mathrm{F} 18^{+}$E. coli by recombinant L. lactis displaying surface anti-F4 ${ }^{+}$and anti-F18 $8^{+} \mathrm{Nb}$, respectively, anchored on the cell wall by protein A. Top panel: (a) positive agglutination of F4 ${ }^{+}$E. coli strain C1065-79 and (b) $\mathrm{F}^{+}$E. coli strains C544-79 by anti-F4 L. lactis (pEXP368; $\alpha \mathrm{F} 4 \mathrm{~V} 4$ on long protein A anchor). In the control tests, (c) no agglutination was seen between E. coli C1065-79 and control L. lactis (pEXP362; $\alpha$ F18 NbFedF6 on long anchor ProtA) or (d) C1065-79 in PBS. Bottom panel: agglutination of F18 ${ }^{+}$E. coli strain by anti-F18 Lactococcus strains, (e) F18 ${ }^{+}$E. coli K514 $\Delta$ fim (pIH120) that contain the gene cluster for F18 fimbriae biogenesis [8] and L. lactis (pEXP362), (f) F18 ${ }^{+}$E. coli K514 $\Delta$ fim (pIH120) and L. lactis (pEXP364 (NbFedF7 on long protein anchor). In the control tests, no agglutination is seen between (g) K514 $\Delta$ fim (pIH120) and non-F18 L. lactis (pEXP368, $\alpha$ F4 V4) or (h) K514 $\Delta$ fim (pIH120) in PBS.

\subsubsection{Effect of Heat Treatment on Viability and Agglutination Capacity}

To test the possibility of using inactivated Lactococcus strains in piglets, the strains were heated at different temperatures and times in a water bath and used in the agglutination test. Successful inactivation was evaluated by colony count (CFU/) $\mathrm{mL}$ of heat-treated samples plated on M17 (Oxoid) agar supplemented with glucose and erythromycin. Complete inactivation with a full agglutinating capacity was achieved at $60^{\circ} \mathrm{C}$ for $20 \mathrm{~min}$. Inactivation at higher temperatures abolished agglutination. The same strains were also treated with 5 $\mathrm{mM}$ dithiothreitol (DTT) for $20 \mathrm{~min}$ to reduce the internal disulfide bond present in the $\mathrm{Nbs}$ anchored to the surface of the recombinant $L$. lactis strains. This treatment abolished agglutination, but the strains remained viable. The results for heat and DTT treatments are summarized in Supplementary Table S3. Non-specific binding was blocked by 3\% bovine serum albumin in PBS.

\subsubsection{Immunofluorescence Microscopy}

To demonstrate the surface display of the anti-FaeG and anti-FedF Nbs by recombinant L. lactis using FITC fluorescence dye, FITC conjugated FaeG and FedF were mixed with the respective anti-FaeG and anti-FedF Nbs expressing L. lactis strains and observed under fluorescence microscope (Figure 4). The anti-FaeG and anti-FedF L. lactis strains specifically recognized the respective antigen conjugates, FaeG-FITC and FedF-FITC, as detected by the positive fluorescence signals. 

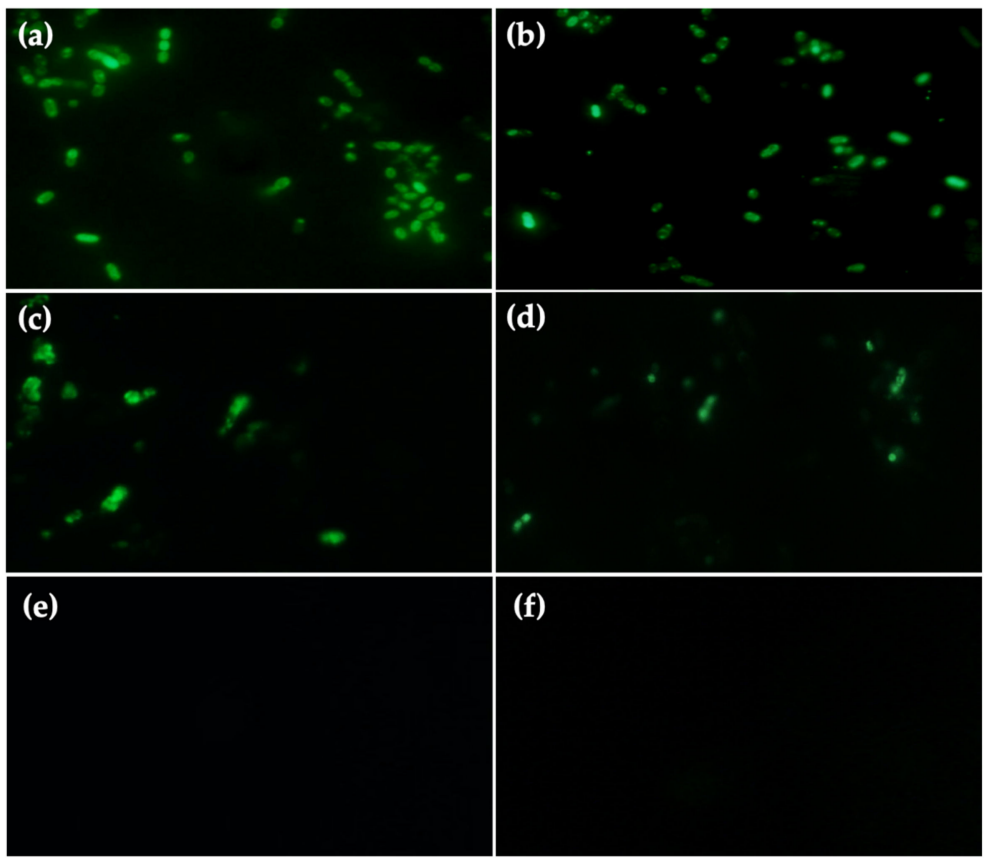

Figure 4. Fluorescence of recombinant L. lactis labeled with (Flourescien-5-isothiocyanate) FITCconjugated antigens. The anti-FaeG and anti-FedF L. lactis strains specifically recognized FITC labeled FaeG and FedF antigens, respectively, as shown by fluorescence microscopy. Slides: (a) anti-FaeG $\mathrm{Nb}$ L. lactis (pEXP368) labeled with FaeG-FITC; (b) anti-FaeG L. lactis (pEXP366) labeled with FaeGFITC; (c) anti-FedF L. lactis (pEXP364) labeled with FedF-FITC; (d) anti-FedF L. lactis (pEXP362) labeled with FedF-FITC; (e) non FaeG/FedF L. lactis (pEXP242) labeled with FaeG-FITC; (f) non FaeG/FedF L. lactis (pEXP242) labeled with FedF-FITC. Image captured at X100 magnification under oil immersion.

\subsubsection{In vitro Adhesion Assay}

The anti-F4 and anti-F18 Nbs expressing L. lactis strains were tested for their inhibitory effect on the respective F4 or F18 E. coli adhesion to piglet intestinal villi in an in vitro adhesion test [22,48].

\section{Lactococcus lactis Expressing anti-FedF Nanobodies}

The anti-FedF L. lactis strains (Table 2) inhibited F18 ${ }^{+}$E. coli (strain 107/86) adhesion to the $\mathrm{F} 18 \mathrm{R}^{+}$piglet villi, as measured by the numbered of $E$. coli cells adhering to the piglet villi. Adhesion inhibition was reversed by pre-incubating anti-FedF $\mathrm{Nb}$ expressing L. lactis with FedF, the tip adhesin of F18 fimbriae (Figures 5a and 6a). Paired t-test showed significant difference in the mean number of $\mathrm{F} 18^{+}$E. coli adhered to the villi, following the adhesion inhibition by anti-FedF L. lactis strains and the reversal of the inhibitory effect by pre-incubating the L. lactis strains with FedF adhesin $(p<0.001)$. The optimal anti-FedF $\mathrm{Nb}$ expressing L. lactis concentration for maximal inhibition was $10^{10}$ cells/.mL Inhibitory effect of L. lactis strains expressing short (83 residues) or long (163 residues) protein A anchor domains was tested in the in vitro assay (Figures $5 b$ and $6 b$ ). All the L. lactis strains with long protA anchor had a strong inhibitory effect on binding of F18+ E. coli that was reversed by pre-incubation of the villi with FedF adhesin $(p<0.001)$, while L. lactis with Short ProtA anchor showed only partial inhibition and adhesion inhibition reversal effects by pre-incubation of the anti-FedF L. lactis strains with purified FedF (Figure 5a) that was significant for NbFedF12 L. lactis strain $(p=0.003)$ and non-significant for NdFedF9 L. lactis strain. 


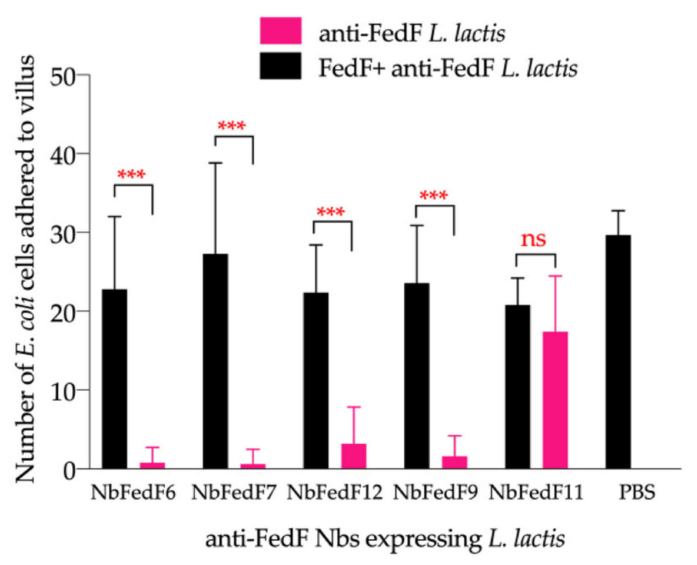

(a)

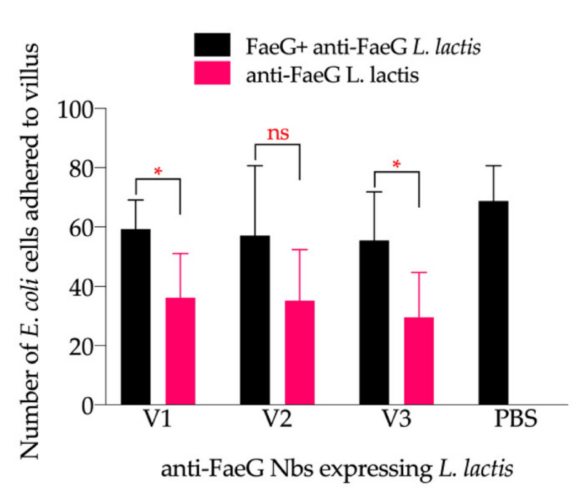

(c)

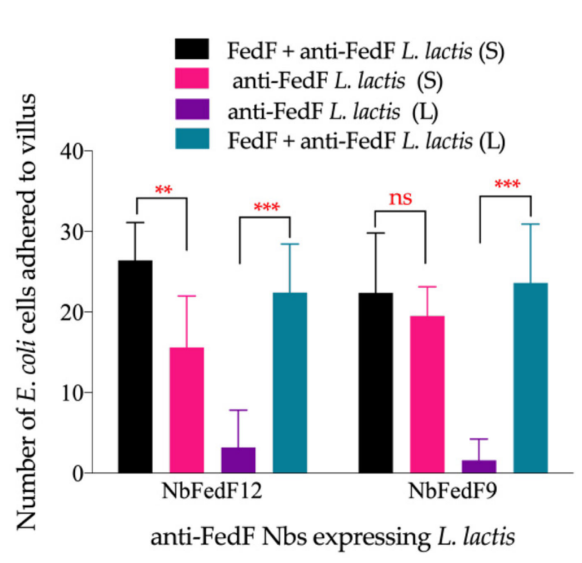

(b)

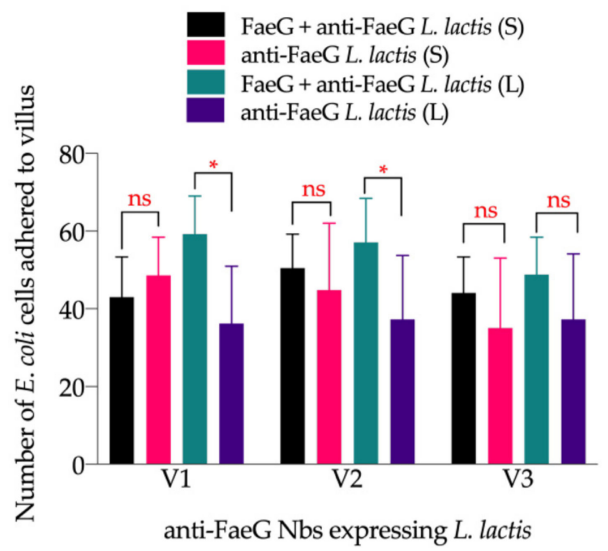

(d)

Figure 5. Inhibition of adherence of the $\mathrm{F}^{+} 8^{+}$and $\mathrm{F} 4^{+}$E. coli strains to prepared piglet microvilli by L. lactis expressing anti-FaeG and anti-FedF Nb. (a) Inhibition of adherence of the F18 ${ }^{+}$E. coli 107/86 to microvilli in vitro by L. lactis expressing anti-FedF $\mathrm{Nbs}$ on the cell surface. All the L. lactis strains showed strong inhibition except for the positive control $\mathrm{Nb} 242$ (non-neutralizing anti-FedF Nb) and E. coli strain 107/86 alone in PBS. Adherence inhibition was reversed by pre-incubating Lactococcus strains with purified FedF protein. (b) Effect of protein A anchor domain length on the inhibition of F18 $8^{+}$E. coli 107/86 adhesion to microvilli by anti-FedF Nb expressing L. lactis. A short ProtA anchor domain (S) resulted in partial inhibition (magenta), whereas a long ProtA (L) caused nearly complete inhibition (purple). This positive adhesion inhibition was reversed by pre-incubation of the anti-FedF L. lactis strains with FedF (black and blue bars). (c) Partial inhibition of F4 ${ }^{+}$ E. coli (C95-72; K88ac) adherence to prepared piglet microvilli by L. lactis expressing surface anti-FaeG Nbs (magenta). This inhibition was reversed by pre-incubation of these Lactococcus strains with purified FaeG (black). All the anti-FaeG L. lactis strains showed partial inhibition. (d) Effect of protein A anchor domain on the inhibition of $\mathrm{F}^{+}$E. coli C95-72 adhesion to microvilli. A short linker (S) did not inhibit adhesion (magenta), whereas a long linker (L) resulted in partial inhibition (purple). Pre-incubation of the anti-FaeG Lactococcus strains with FaeG (black and blue) allowed adhesion. Adherence was measured as counts of $E$. coli cells attached to $250 \mu \mathrm{m}$ villus length. Level of significance: $p>0.05$ (ns), $p \leq 0.05\left(^{*}\right), p \leq 0.002$ $(* *)$, and $p \leq 0.001{ }^{(* * *)}$. 

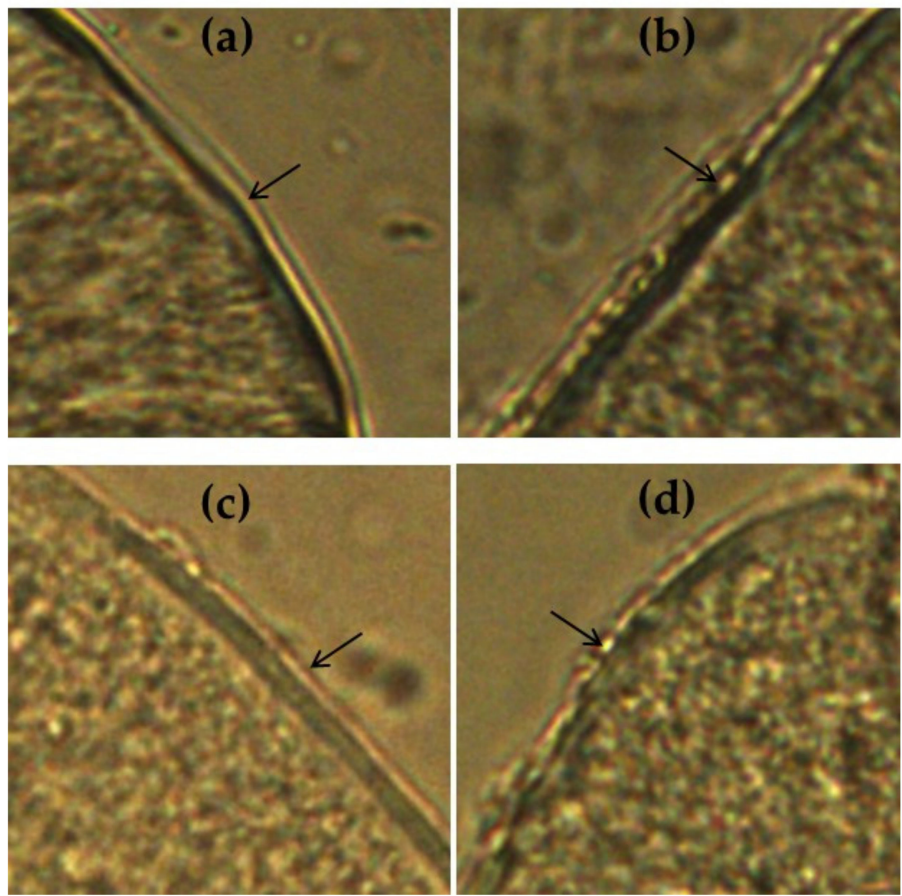

Figure 6. Inhibition of $\mathrm{F} 18^{+}$and $\mathrm{F} 4^{+}$E. coli adhesion to $\mathrm{F} 18 \mathrm{R}^{+}$and $\mathrm{F} 4 \mathrm{R}^{+}$piglet microvilli by anti-FedF or anti-FaeG Nbs expressing L. lactis. (a) Complete adhesion inhibition of F18 ${ }^{+}$E. coli $(107 / 86)$ to F18R ${ }^{+}$microvilli by L. lactis (pEXP362) expressing anti-FedF NbFedF6 on its surface. (b) Inhibitory effect of anti-FedF $\mathrm{Nb}$ expressing L. lactis was blocked by pre-incubating with $50 \mu \mathrm{g}$ of FedF that neutralized surface displayed NbFedF6. (c) Partial inhibition of F4 ${ }^{+}$E. coli (C95-72) adhesion to F4R ${ }^{+}$ microvilli by L. lactis (pEXP368) expressing anti-FaeG Nb V4. (d) Adhesion inhibition was reversed by pre-incubating L. lactis with $50 \mu \mathrm{g}$ of FaeG. The arrow points to the villi surface without bound E. coli $(\mathbf{a}, \mathbf{c})$ and with bound $E$. coli cells $(\mathbf{b}, \mathbf{d})$.

\section{Lactococcus lactis Expressing anti-FaeG Nanobodies}

Anti-FaeG Lactococcus partially inhibited the in vitro adhesion of $\mathrm{F}^{+}$E. coli to the $\mathrm{F}_{4} \mathrm{R}^{+}$villi, and this effect was reversible when the strains were pre-incubated with FaeG, the specific antigen for the Nanobodies displayed by L. lactis strains. Inhibition effect was concentration dependent, with only partial inhibition at $10^{10}$ cells $/ \mathrm{mL}$ for L. lactis (Figures $5 \mathrm{c}$ and $6 \mathrm{~d}$ ). The difference between mean number of $\mathrm{F}^{+} \mathrm{E}$. coli adhered to $\mathrm{F} 4 \mathrm{R}^{+}$villi in the adhesion inhibition and reversal of adhesion inhibition assay was only significant for the V1 $(p=0.004)$ and V3 $(p=0.004)$ L. lactis strains, but it was not significant for V2 L. lactis. Strains expressing short (83 residues) and long (163 residues) ProtA anchor domain sequences were compared in the in vitro assay. The result showed that constructs with a short ProtA anchor domain did not inhibit adhesion, whereas long links resulted in partial agglutination that was reversible on pre-incubation of the Lactococcus strains with purified FaeG. Among the long ProtA anchor L. lactis strains, the mean difference in the number of $\mathrm{F}^{+}$E. coli adhered to the $\mathrm{F}^{+} \mathrm{R}^{+}$villi during the inhibition adhesion and inhibition reversal assays were significant for V1 $(p=0.004)$ and V2 $(p=0.005)$ L. lactis strains but not significant for V3 $(p=0.106)$ L. lactis. The results are shown in Figures $5 d$ and $6 \mathrm{~d}$.

The in vitro adhesion assays thus demonstrated a clear effect of the length of the ProtA anchor domain for the anti-FedF Nbs directed against the tip adhesin FedF (1 adhesin per fimbria) of F18 fimbriae. In comparison, the effect of the length of the ProtA anchor domain inhibition of anti-FaeG Nbs directed against the major subunit and adhesin FaeG (1000 adhesins per fimbria) of F4 fimbriae only resulted in a small difference in the inhibition of adhesion. 


\subsection{In vivo Piglet Challenge Assay}

In a preliminary clinical trial, twenty piglets were weaned at four weeks and randomly divided into three groups for the infection challenge experiment. The $\mathrm{F}^{+}$E. coli strain C95-72 (F4ac) was used in experimental infection challenge. To control $\mathrm{F}^{+}$E. coli infection, the treated group received passive immunization through feeding diet supplemented with a mixture of recombinant anti-FaeG L. lactis (pEXP366(V2) and pEXP368(V4)); the positive control group diet was supplemented with laboratory strain L. lactis MG1363 [46], while the negative control group received no diet supplement (Table 1).

The piglets were monitored for clinical signs of diarrhea daily. The fecal bacterial shedding, weight gain and immune response were measured over a two-week period after which the piglets were sacrificed to assess the individual F4R receptor status by in vitro villous adhesion assay. One piglet from Group 2 and two piglets from Group 3 were found to be F4R negative and were removed from subsequent analysis.

\subsubsection{Weight Gain}

All the piglets were weighed on days 0,7 , and 14 (before sacrificing). Average weight of piglets assigned to the three experimental groups, measured at the three time points, is shown in Figure 7 and Supplementary Table S4. Since the piglets were drawn from different litters and varied in weights on day 0 , percentage weight gain during the 2-week study period was computed to compare growth rate between the different groups. The boxplot in Figure 7 depicts the quartile distribution of the average percent weight for each treatment group. The mean percent weight gain over the study period (day 0-14) was comparable between the three groups. Numerically, the mean percentage weight gain was highest in Group 3 piglets (infected and treated), as shown in Supplementary Table S5, followed by Group 1 (negative control) and least in Group 2 (positive control). Prediction of weight gain using mixed effects logistic regression showed no significant association between treatment group and weight gain (Supplementary Table S6). All interactions between treatment groups and experiment days were not significant.

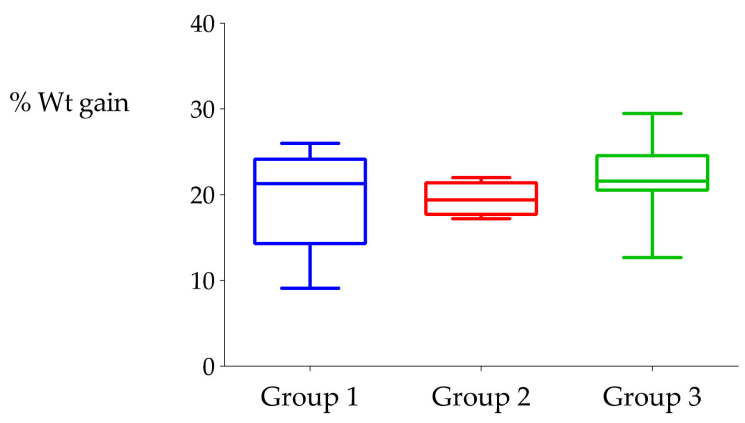

Figure 7. Average weight gain post weaned piglets following challenge with F4 ETEC strain. Group 1, negative group (not infected); Group 2, positive group (not treated and challenged with F4 ${ }^{+}$ETEC); Group 3; test group (treated with anti-FaeG L. lactis added to the feed from day 0 to day 8 and challenged with $\mathrm{F}^{+}$ETEC and). The edges of the boxes are drawn at 25 th and 75 th percentiles, the middle line is the median, and the lines are drawn at $1.5 \mathrm{IQR}$ (inter quartile range).

\subsubsection{Fecal Bacterial Shedding and Diarrhea}

Fecal samples were taken on day $-2,1,3,5,6,7,8,9,10$, and 13 post-challenge. Ten-fold serial dilutions of $10 \mathrm{~g}$ fecal in PBS was plated on MacConkey media for E. coli enumeration. The CFUs per gram of the fecal sample for each piglet was determined and the average for each group computed. The negative control group (Group 1) was used as a reference basal CFUs counts to compare the other groups. The results (Figure 8) showed the infected and treated piglets (Group 3) had lower CFU counts compared to the infected and non-treated piglets (Group 2). Both groups registered the peak CFU counts on day 6 (3 days post-challenge), followed by a progressive decline thereafter. The infected and 
treated piglets (Group 3) however returned to basal level of bacterial shedding 5 days earlier than the infected non-treated piglets (Group 2). An ANOVA test comparing the bacterial shedding between groups concluded that the mean CFU was not significantly different for all groups $(F=2.92, p<0.071)$. None of the piglets in Group 1 (non-infected) and Group 3 (infected and treated) developed clinical diarrhea during the study period. Among the piglets in Group 2 (infected and not treated), two piglets (out of 5) developed clinical diarrhea starting day 3 and 4 post challenge. The diarrhea lasted for 2-3 days and was self-limiting. Mixed effects logistic regression analysis of Log10 CFU/g fecal bacteria shedding by experimental piglets, with treatment and experiment days as fixed factors and animal ID as random factor, estimated 0.24 and 0.28 logs decrease in fecal bacterial shedding, by groups 2 and 3 respectively, although these decreases were not significant. Analysis of the interaction between treatment groups and experiment day estimated a $>1$ $\log$ increase in fecal bacterial shedding in group 2 on days 5-10 compared to reference group 1 , and this increase was significant $(p<0.05)$. In group 3 , significant increase in fecal bacterial shedding $(p<0.05)$ was observed on days $3-6$, but a $>1$ log increase was estimated on day 5 only (Supplementary Table S7).

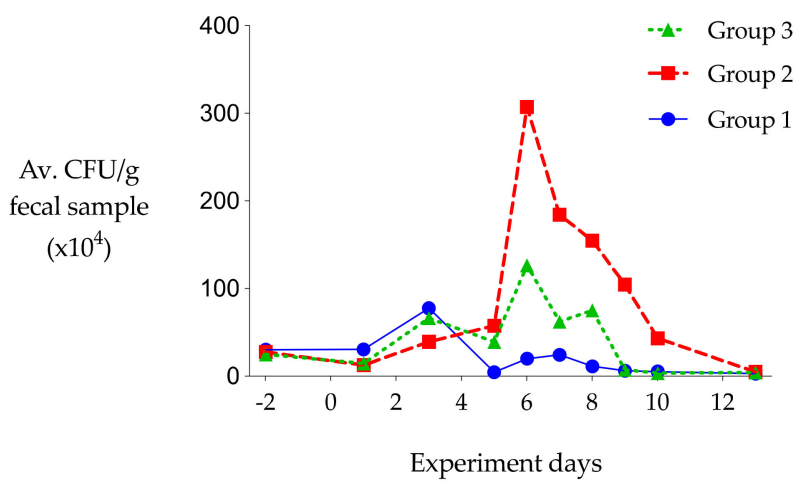

Figure 8. Bacterial load in fecal samples measured as CFU. The piglets were challenged on days 2 and 3. Peak fecal bacterial load was recorded on day 6 in groups 2 and 3, after which there was a progressive decline. Group 2 had lower CFU counts than group 3 and resolved quickly to the basal level (Group 1) on day 9. Group 1, negative control group (not infected); Group 2, positive group (infected and not treated); Group 3; test group (infected with $\mathrm{F}^{+}$ETEC and anti-FaeG Nanobodies on the surface of L. lactis added to the meals from day 0 to day 8 ).

\subsubsection{Immune Response}

Blood samples were taken on days 0,7 , and 14, and a change in IgG levels of these blood samples were determined using an anti-FaeG ELISA (Figure 9). Immune response was measured as OD (optical density) in the ELISA method. The calculated inter- and intra-assay coefficient of variation $(\mathrm{CV})$ were $5.7 \%$ and $6.2 \%$ respectively. Increased antiFaeG IgG level was observed in the infected and treated piglets (Group 3) compared to the challenged and non-treated piglets (Group 2), while the negative control group had negligible change in the IgG levels (Figure 9). Prediction of immune response using mixed effects logistic regression estimates showed no significant effects of treatment group and experiment days on immune response, although the analysis indicated a lower and increased immune response in groups 2 and 3, respectively. A similar pattern was observed for experiment days 7 and 14. However, analysis of the interaction between treatment group and experiment day estimated a significant increased immune response for group 3 on day $14(p<0.001)$ (Supplementary Table S8). 


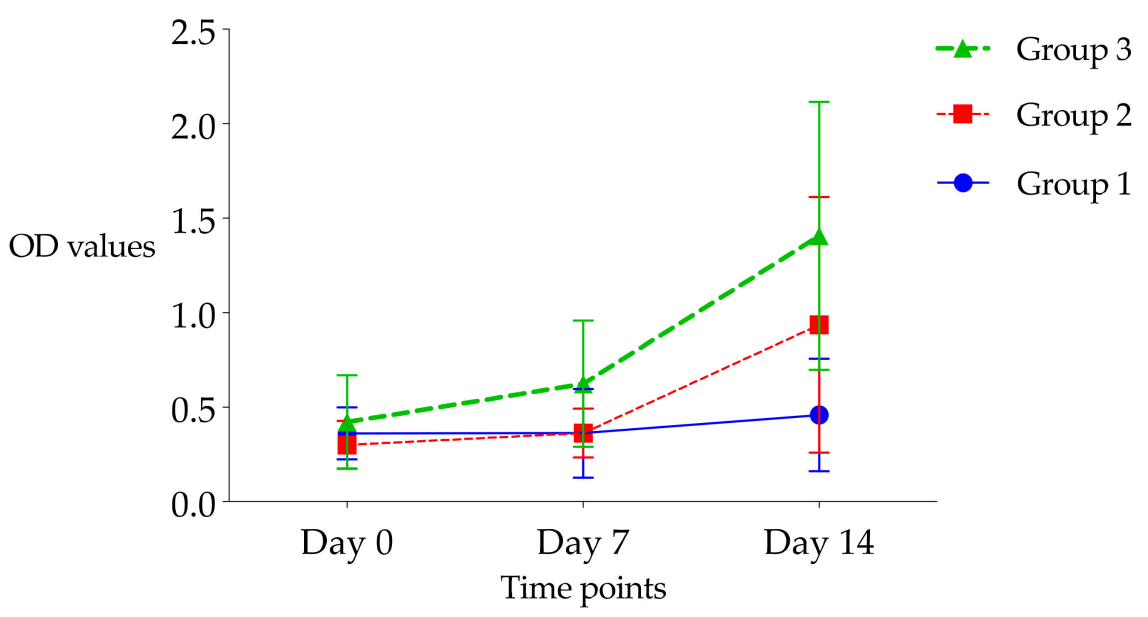

Figure 9. Mean IgG level in sera samples taken on days 0,7 , and 14 . IgG level was measured using an anti-FaeG ELISA and depicted as optical density (OD) at $450 \mathrm{~nm}$. The error bars indicate standard deviation. Group 1, negative group (not infected); Group 2, positive group (infected and not treated); Group 3; test group (infected with F4 ETEC and anti-F4 L. lactis added to the meals from day 0 to day 8).

\section{Discussion}

Despite several research efforts to develop vaccines against post-weaning diarrhea in piglets, there is still no effective vaccine available on the market. Previous researches focusing on oral immunization with live or subunit vaccines failed to give protective immunity, due to the limited time available for the piglets to mount an immune response after weaning, given the fact that lactogenic immunity neutralizes vaccines during the suckling period [15]. Passive immunization is currently considered the most viable option in preventing post-weaning diarrhea in piglets [37]. The major challenge is however to design a cost-effective method for mass production for commercial application. L. lactis is a normal resident of the intestinal flora and is a suitable system due to the GRAS (generally regarded as safe) status $[27,29,53]$. In this study, we grafted the llama Heavy chain antibody binding domains (Nanobodies or $\mathrm{Nbs}$ ) on protein A anchor domain derived from S. aureus for surface display on L. lactis through covalent linkage to the cell wall. These L. lactis producing the fused $\mathrm{Nb}$ on ProtA anchor domain were subsequently used for passive immunization.

Successful Nanobody display in active form was evaluated in vitro by slide agglutination, immunofluorescence, flow cytometry, and villous adhesion inhibition assay. All tests confirmed correct display in biologically active form. The recombinant $L$. lactis strains specifically recognized F4 or F18 E. coli strains in a slide agglutination assay and abrogated bacterial adhesion to F4 or F18 receptor positive villous preparations in vitro. Whereas adhesion inhibition of F18 strains was complete, the F4 strains were only partially inhibited possibly due to the fact that the targeted FaeG adhesin subunit is also the major fimbrial subunit that exists in multiple copies [54]. FedF tip adhesin of F18 strains on the other hand is a single copy fimbrial tip adhesin that can be easily neutralized. We compared the inhibitory effect of the Nbs with varied ProtA anchor domain length and could demonstrate that a longer linker peptide improved the inhibition of villous adhesion for both anti-F4 and anti-F18 producing L. lactis strains. This could be attributed to the improved degree of mobility and increased display exposure outside the bacterial surface that enables a wider sweep area for the attached Nanobodies to locate and bind their cognate receptors on E. coli cells.

In a challenge experiment, only the anti-FaeG L. lactis was tested on the basis of the known experimental results when expressed in the plant system [36]. Anti-FedF L. lactis will be tested in future challenge experiments. A minimum dosage of Lactococcus $\left(10^{10}\right.$ cells $/ 100$ $\mathrm{g}$ of feed for of the three three anti-FaeG Nanobodies) was used on the basis of inhibitory concentrations in the in vitro assay. We were able to culture a low concentration of the 
anti-FaeG L. lactis in the fecal samples of the piglets from the treated group, indicating these strains could remain viable through the gastrointestinal tract. Since the L. lactis strains retained activity following heat inactivation, future experiments should test the protective effect of heat inactivated strains in vivo to address any concerns regarding the use of live organisms. Such experiments should evaluate several dosage regimes.

Results of the challenge experiment showed that passive immunization by in-fed addition of the recombinant Lactococcus strains protected the piglets against F4 ETEC colonization, as shown by reduced fecal bacterial shedding following challenge in the treated group compared to the non-treated. The slightly elevated bacterial counts in the treated group were also quickly reduced to the basal level with 6 days after challenge. In addition, none of the challenged and treated piglets developed diarrhea, while two in five piglets in the challenged and non-treated group had diarrhea, indicating the potential of anti-FaeG L. lactis to protect piglets against post-weaning diarrhea. This confirms the results previously reported using the same Nanobodies expressed in Arabidopsis thaliana [37]. However, use of a microbial system is a much more viable option, due to the ease and cost effectiveness of scaling up production for commercial application. There was a marginal improved weight gain in the treated group compared to the positive control, indicating that the reduced bacterial load impacted positively on the growth rate. Since the piglets varied in the weaning weight, percentage weight gain was a preferred method for comparison. Future experiments should use piglets of similar weight for a more accurate growth rate comparison. The result of the ELISA showed that the low anti-FaeG IgG level (OD values) among the non-infected piglets (Group 1) remained low throughout the follow up period; this was an expected outcome in the absence of F4-positive ETEC challenge. Among the other groups, increased OD values were observed in the infected and treated piglets (Group 3) compared to the challenged and non-treated piglets (Group 2). Group 2 piglets were untreated but challenged, resulting in massive shedding of the F4-positive ETEC at days 6, 7, and 8, while Group 3 (treated and challenged) only shed very low levels of the F4-positive ETEC strain. We hypothesize that the F4 immunity in the piglets was primed during the suckling period, as shown by the high OD values in all the three groups on day 0 . The low amount of challenge F4+ ETEC boosted the immune response in both Group 2 and 3 piglets. However, the immune response in Group 2 piglets was initially suppressed while shedding large numbers of ETEC bacteria but increased later when ETEC shedding reduced. Group 3 had a significantly higher OD value on day 14 compared to the other groups, which could be explained by the ETEC immune boost that was not impeded since the L. lactis added in diet was protective against colonization and limited ETEC shedding. This hypothesis is supported by the observed low OD values in the F4R negative piglets in Groups 2 and 3, that remain at the levels observed for the non-infected group. It is already known for a long time that the immune response to oral administered F4 fimbriae is F4R receptor-dependent [55]. Hence, F4R-negative piglets will not develop an immune response. On the other hand, an increased immunity is a desired outcome that ensures protection following withdrawal of the probiotic supplement. Additionally, the improved immune response in the treated group could also be possibly explained by non-specific immunomodulatory effect of lactic acid bacteria [56]. At weaning, the microbial population structure was shown to change from predominantly Lactobacilli species at neonatal stage to emergence of Clostridia and E. coli during the early weaning stage [57]. Addition of L. lactis at weaning possibly stabilized the gut microbiota and improved immune response [58].

At the end of the challenge experiment, three piglets from two treatment groups were shown to be F4R negative and excluded from the analysis. Prescreening for the F4R receptor is not possible, as only postmortem in vitro adhesion assay can ultimately determine the F4R status of piglets. Genotypic detection of mucin4 genotype polymorphism have been considered a possible prescreening method for F4R status but is not an accurate predictor for the susceptibility phenotype [59]. 


\section{Conclusions}

Passive immunization of piglets with recombinant Lactococcus strains could be a costeffective alternative to control post-weaning diarrhea and edema disease. Therefore, we constructed recombinant L. lactis displaying anti-FaeG (F4 fimbriae) Nbs or anti-FedF (F18 fimbriae) Nbs on the bacterial surface. The presence of the Nbs on the surface of recombinant $L$. lactis was shown by agglutination and immunofluorescence microscopy. These recombinant $L$. lactis also inhibit the in vitro adhesion of the corresponding ETEC bacteria to microvilli. The recombinant $L$. lactis expressing the anti-FaeG (F4 fimbriae) $\mathrm{Nbs}$ was also used to evaluate the in vivo protection of weaned piglets against F4 ETEC infections. We could demonstrate the potential use of recombinant Lactococcus strains for passive immunization against F4 ETEC infections causing piglet post-weaning diarrhea. If the anti-FedF Nbs expressing L. lactis strains are also protective in vivo, combining both strains in feed would offer complete protection against ETEC and STEC infections during the weaning period.

Supplementary Materials: The following are available online at https:/ / www.mdpi.com/2077-047 2/11/3/186/s1, Figure S1: Sequence alignment of the fusion protein (Signal peptide-FaeG NanobodyProtA) expressed in Lactococcus lactis, Figure S2: Sequence alignment of the fusion protein (Signal peptide-FedF Nanobody-ProtA) expressed in Lactococcus lactis, Table S1: F18 L. lactis agglutination assay, Table S2: F4 L. lactis agglutination assay, Table S3: Effect of heat treatment on viability $(\mathrm{CFSs} / \mathrm{mL})$ and agglutination properties of L. lactis strains, Table S4: Average weight of piglets assigned to the 3 experimental groups, Table S5: Meant percent weight gain between day 0-14, Table S6: Mixed effects logistic regression estimates of weight gain in experimental piglets with treatment and experiment days as fixed factors, and animal ID as random factor, Table S7: Mixed effects logistic regression estimates of $\log 10 \mathrm{CFU} / \mathrm{g}$ fecal bacteria shedding by experimental piglets, with treatment and experiment days as fixed factors and animal ID as random factor, Table S8: Mixed effects logistic regression estimates of immune response in experimental piglets with treatment and experiment days as fixed factors, and animal ID as random factor.

Author Contributions: H.D.G. conceived the study. E.O. made the recombinant L. lactic strains. E.O. and K.M. carried out the in vitro characterization of the recombinant $L$. lactis strains and analyzed the results. E.O. and J.E. did the in vivo pig studies and the analysis of the challenge data. E.O. and H.D.G. interpreted all the results and wrote the manuscript. All authors have read and agreed to the published version of the manuscript.

Funding: This research was funded by VLIR-UOS (Vlaamse Interuniversitaire Raad) under the Interuniversity Development Cooperation program (Belgium).

Institutional Review Board Statement: "The study was conducted according to the guidelines of the Declaration of Helsinki and approved by the Institutional Review Board (or Ethics Committee) of the Uganda National Council for Science and Technology (UNCST) on 16 April 2013; Approval \#HS 1361)."

Informed Consent Statement: Not applicable.

Data Availability Statement: Not applicable.

Acknowledgments: The authors thank F. Deboeck for excellent technical assistance in constructing the Gateway shuttle vectors. We also thank Eric Cox (Ghent University) for the intestinal villi from the mid-jejunum of four-week-old piglets, positive for the F4 or F18 receptor. Finally, we thank the School of Veterinary Medicine, Makerere University, for providing animal housing facilities and care for the piglets.

Conflicts of Interest: The authors declare no conflict of interest. 
Appendix A Detailed Experiment Procedures

Appendix A.1 Primers Sequences

\begin{tabular}{|c|c|}
\hline Primer Name & Nucleotide Sequence $\left(5^{\prime}-3^{\prime}\right)$ \\
\hline $\operatorname{coc} 1$ & $\begin{array}{l}\text { GGGGACAAGTTTGTACAAAAAAGCAGGCTGAATTCAGAAAGGAGA } \\
\text { TATACGCATGAAAAAAAAGATTATCTCAGCTATTTTAATG }\end{array}$ \\
\hline $\operatorname{coc} 2$ & $\begin{array}{c}\text { AGATTATCTCAGCTATTTTAATGTCTACAGTGATACTTTCTGCTGCAGC } \\
\text { CCCGTTGTCAGGTGTTTACGCTCAGGTGCAGCTGCAGGAGTCTG }\end{array}$ \\
\hline $\operatorname{coc} 3$ & $\begin{array}{l}\text { CTATTGGGGCCAGGGGACCCAGGTCACCGTCTCCTCAGGAC } \\
\text { CAAAAGAGGAAGACAATAACAAGCCT }\end{array}$ \\
\hline $\operatorname{coc} 4$ & $\begin{array}{c}\text { AGGCTTGTTATTGTCTTCCTCTTTTGGTCCTGAGGAGACGG } \\
\text { TGACCTGGGTCCCCTGGCCCCAATAG }\end{array}$ \\
\hline $\operatorname{coc} 5$ & $\begin{array}{l}\text { GGGGACCACTTTGTACAAGAAAGCTGGGTAAAGCTTTTTATAGTTCG } \\
\text { CGACGACGTCCAGCTAATA }\end{array}$ \\
\hline $\operatorname{coc} 19$ & $\begin{array}{l}\text { CTTTGTACAAAAAAGCAGGCTGAATTCAGAAAGGAGATATACGCATG } \\
\text { AAAAAAAAGATTATCTCAGCTATTTTAATG }\end{array}$ \\
\hline $\operatorname{coc} 20$ & GACCCAGGTCACCGTCTCCTCAGGACCAAAAGAG \\
\hline Aida9 & GCGAAATTAATACGACTCACTATA \\
\hline pETrev & GGTTATGCTAGTTATTGCTCAGCG \\
\hline pTRKH8 & CTAATAAAGCCGTAAGGAGACGGGTTCA \\
\hline pTRKH9 & CССATCCTAACGGCCACG CATATG \\
\hline
\end{tabular}

Appendix A.2 Construction of Nb-ProtA Recombinant Fusion Genes

The $\mathrm{Nb}$ coding sequences were amplified from plasmids containing the inserts. These Nbs were generated in our laboratory and are well characterized [40,41]. The anchor domain of ProtA were amplified from total genomic DNA of S. aureus NTC8325 [45] using primers pair $\operatorname{coc} 3$ and $\operatorname{coc} 5$. The $\mathrm{Nb}$ coding sequences were amplified using primers $\operatorname{coc} 2$ and coc4 from miniprep DNA. The primers $\operatorname{coc} 4$ and $\operatorname{coc} 3$ were constructed with the respective $3^{\prime}$ and $5^{\prime}$ ends extension complementary to a segment of the $\mathrm{Nb}$ and Prot $\mathrm{A}$ sequences, and $\operatorname{coc} 2$ had $5^{\prime}$ extension of Usp45 signal sequence. $\mathrm{Nb}$ and ProtA PCR fragments were joined in an overlap PCR reaction using the primer pair coc1 and coc5. Primers coc1 and coc5 had, respectively, a $5^{\prime}$ and $3^{\prime}$ extension of attB sequence of the gateway system. The primer sequences are outlined in the table above.

All PCR reaction volumes were $50 \mu \mathrm{L}$ PCR: $5 \mu \mathrm{L}$ 10x ExTakara buffer, $4 \mu \mathrm{L} 2.5 \mathrm{mM}$ dNTPs mix, $1 \mu \mathrm{L}$ each $20 \mu \mathrm{M}$ primers, $0.2 \mu \mathrm{L}$ ExTakara DNA polymerase, $10 \mu \mathrm{L}$ DNA template, and $14.4 \mu \mathrm{L} \mathrm{dH_{2 }} \mathrm{O}$. The cycling parameters were: initial denaturation at $94{ }^{\circ} \mathrm{C}$ for $55 \mathrm{sec}$, denaturation at $94^{\circ} \mathrm{C}$ for $10 \mathrm{sec}$, annealing at $55^{\circ} \mathrm{C}$ for $30 \mathrm{sec}$, extension at $68^{\circ} \mathrm{C}$ for $2 \mathrm{~min}$, and final extension at $72{ }^{\circ} \mathrm{C}$ for $7 \mathrm{~min}$.

\section{Appendix A.3 Adapting pTRKH3 Vector to the Gateway System}

To adapt the pTRKH3 to the Gateway system, the vector was restricted by EcoRI and ApaLI restrictions enzymes in two separate reactions and the ends of the linear plasmid blunted by T4 polymerase. The reaction mix comprised of $15 \mu \mathrm{L}$ restricted plasmid DNA, $2 \mu \mathrm{L}$ dNTPs ( $2.5 \mathrm{mM}$ each), $2 \mu \mathrm{L} \mathrm{H} \mathrm{H}_{2} \mathrm{O}$ and $1 \mu \mathrm{L}$ T4 DNA polymerase. The pDEST14 cassette was amplified with the primers Aida9 and pETrev (same PCR protocol as above; primer sequences are outlined in the table above), and the PCR fragment was blunted with T4 polymerase for subsequent ligation with a linearized and blunted pTRKH3 vector, using Boehringer Quick ligation kit. The blunting reaction mix comprised of $15 \mu \mathrm{L}$ Qiaquick purified PCR fragment, $2 \mu \mathrm{L}$ of 10x restriction buffer or 10x T4 DNA polymerase buffer, $2 \mu \mathrm{L}$ of dNTPs (2.5 mM each), and $1 \mu \mathrm{L}$ T4 DNA polymerase. The blunting reactions were incubated at $15{ }^{\circ} \mathrm{C}$ for $15 \mathrm{~min}$ and inactivated at $65^{\circ} \mathrm{C}$ for $10 \mathrm{~min}$. The subsequent ligation 
reaction (Boehringer Rapid Ligation kit) comprised of $5.5 \mu \mathrm{L}$ ApaLI-linearized vector (blunted), $2.5 \mu \mathrm{L}$ pDEST14 cassette PCR fragment (blunted), $2.0 \mu \mathrm{L}$ 5X DNA ligation buffer, $10 \mu \mathrm{L} 2 \mathrm{X}$ T4 DNA ligation buffer and 1.0 $\mu \mathrm{L}$ T4 DNA ligase. The reaction was incubated at $16{ }^{\circ} \mathrm{C}$ for $5 \mathrm{~min}$, and the product was immediately transformed into CSH50 gyrA462 cells $[60,61]$ and plated on LB plates supplemented with chloramphenicol. Miniprep DNA prepared (Qiaquick Miniprep DNA kit) from selected transformed colonies were analyzed for the presence and nature of the inserts by EcoRI and HindIII double digest, and agarose gel electrophoresis.

\section{Appendix A.4 Gibson Assembly Exchange Reaction}

Initial screening and sequencing of pENTR clones showed variability in the ProtA sequence length. To standardize ProtA length, one long and short $\mathrm{Nb}$ (V1)-ProtA fusion gene ( $p E N T R-V 1 L$ and pENTR-V1S) were selected and confirmed by sequencing. The other Nbs against F4 (V2, V3, V4), and F18 (the inhibiting Nb's NbFedF6, NbFedF7, NbFedF9 or NbFedF12 and the non-inhibiting $\mathrm{Nb} \mathrm{NbFedF11)} \mathrm{were} \mathrm{exchanged} \mathrm{with} \mathrm{V1} \mathrm{of} \mathrm{pENTR-}$ V1L and pENTR-V1S in a Gibson assembly reaction [62] to give constructs of uniform ProtA length. Briefly, the $\mathrm{Nb}$ genes were amplified sequentially with $\operatorname{coc} 2 / \operatorname{coc} 20$ and $\operatorname{coc} 19 / \operatorname{coc} 20$ (primer sequences outlined in the table above) to introduce the Usp45 signal peptide and end homology with EcoR1/BstEII restricted pENTR-V1 constructs. The fusion genes and linearized vector were joined in a Gibson Assembly ${ }^{\circledR}$ reaction according to the manufacture's manual. The reaction products were transformed into $\mathrm{DH} 5 \alpha \mathrm{CaCl}_{2}-$ competent cells and correct insert confirmed by colony PCR and sequencing. Confirmed $\mathrm{Nb}$-ProtA constructs were transferred into destination vector $\mathrm{pHD669}$ in a LR reaction (as previously described). The LR product was then transformed into $\mathrm{DH} 5 \alpha \mathrm{CaCl}_{2}$-competent cells and selected on LB media supplemented with tetracycline and confirmed by PCR and sequencing primer pair pTRKH8 and pTRKH9 (primer sequences are outlined in the table above). Miniprep DNA prepared from expression clones in DH5 $\alpha$ were used to transform electro-competent L. lactis MG1363 [44]. Electroporation conditions were conducted at $2.5 \mathrm{kV}, 200 \Omega$, and $25 \mu \mathrm{F}$. Positive clones were selected on M17 media supplemented with $0.5 \%$ glucose and $10 \mu \mathrm{g} / \mathrm{mL}$ erythromycin and confirmed by colony PCR.

\section{References}

1. Gyles, C.L. Escherichia coli in Domestic Animals and Humans; CAB International: Wallingford, Oxon, UK, 1994.

2. Luppi, A.; Gibellini, M.; Gin, T.; Vangroenweghe, F.; Vandenbroucke, V.; Bauerfeind, R.; Bonilauri, P.; Labarque, G.; Hidalgo, Á. Prevalence of virulence factors in enterotoxigenic Escherichia coli isolated from pigs with post-weaning diarrhoea in Europe. Porc. Health Manag. 2016, 2, 20. [CrossRef] [PubMed]

3. Duan, Q.; Yao, F.; Zhu, G. Major virulence factors of enterotoxigenic Escherichia coli in pigs. Ann. Microbiol. 2012, 62, 7-14. [CrossRef]

4. Ho, W.S.; Tan, L.K.; Ooi, P.T.; Yeo, C.C.; Thong, K.L. Prevalence and characterization of verotoxigenic-Escherichia coli isolates from pigs in Malaysia. BMC Vet. Res. 2013, 9, 1-7. [CrossRef]

5. Mainil, J. Escherichia coli virulence factors. Vet. Immunol. Immunopathol. 2013, 152, 2-12. [CrossRef]

6. Toledo, A.; Gómez, D.; Cruz, C.; Carreón, R.; López, J.; Giono, S.; Castro, A.M. Prevalence of virulence genes in Escherichia coli strains isolated from piglets in the suckling and weaning period in Mexico. J. Med. Microbiol. 2012, 61, 148-156. [CrossRef]

7. Zhang, W.; Zhao, M.; Ruesch, L.; Omot, A.; Francis, D. Prevalence of virulence genes in Escherichia coli strains recently isolated from young pigs with diarrhea in the US. Vet. Microbiol. 2007, 123, 145-152. [CrossRef] [PubMed]

8. Imberechts, H.; De Greve, H.; Schlicker, C.; Bouchet, H.; Pohl, P.; Charlier, G.; Bertschinger, H.; Wild, P.; Vandekerckhove, J.; Van Damme, J.; et al. Characterization of F107 fimbriae of Escherichia coli 107/86, which causes edema disease in pigs, and nucleotide sequence of the F107 major fimbrial subunit gene, fedA. Infect. Immun. 1992, 60, 1963-1971. [CrossRef] [PubMed]

9. Bergan, J.; Dyve Lingelem, A.B.; Simm, R.; Skotland, T.; Sandvig, K. Shiga toxins. Toxicon 2012, 60, 1085-1107. [CrossRef] [PubMed]

10. Fleckenstein, J.M.; Hardwidge, P.R.; Munson, G.P.; Rasko, D.A.; Sommerfelt, H.; Steinsland, H. Molecular mechanisms of enterotoxigenic Escherichia coli infection. Microbes Infect. 2010, 12, 89-98. [CrossRef]

11. Ou, B.; Jiang, B.; Jin, D.; Yang, Y.; Zhang, M.; Zhang, D.; Zhao, H.; Xu, M.; Song, H.; Wu, W.; et al. Engineered recombinant Escherichia coli probiotic strains integrated with $\mathrm{f} 4$ and $\mathrm{f} 18$ fimbriae cluster genes in the chromosome and their assessment of immunogenic efficacy in vivo. ACS Synth. Biol. 2020, 9, 412-426. [CrossRef] 
12. Verdonck, F.; Cox, E.; Van Gog, K.; Van der Stede, Y.; Duchateau, L.; Deprez, P.; Goddeeris, B.M. Different kinetic of antibody responses following infection of newly weaned pigs with an F4 enterotoxigenic Escherichia coli strain or an F18 verotoxigenic Escherichia coli strain. Vaccine 2002, 20, 2995-3004. [CrossRef]

13. Cox, E.; Van Der Stede, Y.; Verdonck, F.; Snoeck, V.; Van Den Broeck, W.; Goddeeris, B. Oral immunisation of pigs with fimbrial antigens of enterotoxigenic E. coli: An interesting model to study mucosal immune mechanisms. Vet. Immuno. Immunopathol. 2002, 87, 287-290. [CrossRef]

14. Moon, H.W.; Bunn, T.O. Vaccines for preventing enterotoxigenic Escherichia coli infections in farm animals. Vaccine 1993, 11, 213-220. [CrossRef]

15. Melkebeek, V.; Goddeeris, B.M.; Cox, E. ETEC vaccination in pigs. Vet. Immunol. Immunopathol. 2013, 152, 37-42. [CrossRef]

16. Verdonck, F.; Cox, E.; Van Der Stede, Y.; Goddeeris, B.M. Oral immunization of piglets with recombinant F4 fimbrial adhesin FaeG monomers induces a mucosal and systemic F4-specific immune response. Vaccine 2004, 22, 4291-4299. [CrossRef]

17. Van Den Broeck, W.; Cox, E.; Goddeeris, B.M. Induction of immune responses in pigs following oral administration of purified F4 fimbriae. Vaccine 1999, 17, 2020-2029. [CrossRef]

18. Verdonck, F.; Tiels, P.; van Gog, K.; Goddeeris, B.M.; Lycke, N.; Clements, J.; Cox, E. Mucosal immunization of piglets with purified F18 fimbriae does not protect against F18+ Escherichia coli infection. Vet. Immunol. Immunopathol. 2007, 120, 69-79. [CrossRef]

19. Lee, W.J.; Cha, S.; Shin, M.; Jung, M.; Islam, M.A.; Cho, C.S.; Yoo, H.S. Efficacy of thiolated eudragit microspheres as an oral vaccine delivery system to induce mucosal immunity against enterotoxigenic Escherichia coli in mice. Eur. J. Pharm. Biopharm. 2012, 81, 43-48. [CrossRef]

20. Snoeck, V.; Huyghebaert, N.; Cox, E.; Vermeire, A.; Vancaeneghem, S.; Remon, J.P.; Goddeeris, B.M. Enteric-coated pellets of F4 fimbriae for oral vaccination of suckling piglets against enterotoxigenic Escherichia coli infections. Vet. Immunol. Immunopathol. 2003, 96, 219-227. [CrossRef]

21. Felder, C.B.; Vorlaender, N.; Gander, B.; Merkle, H.P.; Bertschinger, H.U. Microencapsulated enterotoxigenic Escherichia coli and detached fimbriae for peroral vaccination of pigs. Vaccine 2000, 19, 706-715. [CrossRef]

22. Coddens, A.; Verdonck, F.; Tiels, P.; Rasschaert, K.; Goddeeris, B.M.; Cox, E. The age-dependent expression of the F18+ E. coli receptor on porcine gut epithelial cells is positively correlated with the presence of histo-blood group antigens. Vet. Microbiol. 2007, 122, 332-341. [CrossRef]

23. Owusu-Asiedu, A.; Nyachoti, C.M.; Marquardt, R.R. Response of early-weaned pigs to an enterotoxigenic Escherichia coli (K88) challenge when fed diets containing spray-dried porcine plasma or pea protein isolate plus egg yolk antibody, zinc oxide, fumaric acid, or antibiotic. J. Anim. Sci. 2003, 81, 1790-1798. [CrossRef]

24. Niewold, T.A.; van Dijk, A.J.; Geenen, P.L.; Roodink, H.; Margry, R.; van der Meulen, J. Dietary specific antibodies in spray-dried immune plasma prevent enterotoxigenic Escherichia coli F4 (ETEC) post weaning diarrhoea in piglets. Vet. Microbiol. 2007, 124, 362-369. [CrossRef] [PubMed]

25. Jȩdrejek, D.; Levic, J.; Wallace, J.; Oleszek, W. Animal by-products for feed: Characteristics, European regulatory framework, and potential impacts on human and animal health and the environment. J. Anim. Feed Sci. 2016, 25, 189-202. [CrossRef]

26. Hu, C.X.; Xu, Z.R.; Li, W.F.; Dong, N.; Lu, P.; Fu, L.L. Secretory expression of K88 (F4) fimbrial adhesin FaeG by recombinant Lactococcus lactis for oral vaccination and its protective immune response in mice. Biotechnol. Lett. 2009, 31, 991-997. [CrossRef]

27. Wei, C.H.; Liu, J.K.; Hou, X.L.; Yu, L.Y.; Lee, J.S.; Kim, C.J. Immunogenicity and protective efficacy of orally or intranasally administered recombinant Lactobacillus casei expressing ETEC K99. Vaccine 2010, 28, 4113-4118. [CrossRef]

28. Andersen, K.K.; Marcotte, H.; Álvarez, B.; Boyaka, P.N.; Hammarström, L. In situ gastrointestinal protection against anthrax edema toxin by single-chain antibody fragment producing lactobacilli. BMC Biotechnol. 2011, 11, 1-11. [CrossRef] [PubMed]

29. Günaydin, G.; Zhang, R.; Hammarström, L.; Marcotte, H. Engineered Lactobacillus rhamnosus GG expressing IgG-binding domains of protein G: Capture of hyperimmune bovine colostrum antibodies and protection against diarrhea in a mouse pup rotavirus infection model. Vaccine 2014, 32, 470-477. [CrossRef]

30. De Greve, H.; Virdi, V.; Bakshi, S.; Depicker, A. Simplified monomeric VHH-Fc antibodies provide new opportunities for passive immunization. Curr. Opin. Biotechnol. 2020, 61, 96-101. [CrossRef]

31. Hamers-Casterman, C.; Atarhouch, T.; Muyldermans, S.; Robinson, G.; Hammers, C.; Songa, E.B.; Bendahman, N.; Hammers, R. Naturally occurring antibodies devoid of light chains. Nature 1993, 363, 446-448. [CrossRef] [PubMed]

32. De Genst, E.; Saerens, D.; Muyldermans, S.; Conrath, K. Antibody repertoire development in camelids. Dev. Comp. Immunol. 2006, 30, 187-198. [CrossRef] [PubMed]

33. Pinto Torres, J.E.; Goossens, J.; Ding, J.; Li, Z.; Lu, S.; Vertommen, D.; Naniima, P.; Chen, R.; Muyldermans, S.; Sterckx, Y.G.J.; et al. Development of a Nanobody-based lateral flow assay to detect active Trypanosoma congolense infections. Sci. Rep. 2018, 8, 1-15. [CrossRef]

34. He, Y.; Ren, Y.; Guo, B.; Yang, Y.; Ji, Y.; Zhang, D.; Wang, J.; Wang, Y.; Wang, H. Development of a specific nanobody and its application in rapid and selective determination of Salmonella enteritidis in milk. Food Chem. 2020, 310, 125942. [CrossRef] [PubMed]

35. Virdi, V.; Palaci, J.; Laukens, B.; Ryckaert, S.; Cox, E.; Vanderbeke, E.; Depicker, A.; Callewaert, N. Yeast-secreted, dried and food-admixed monomeric IgA prevents gastrointestinal infection in a piglet model. Nat. Biotechnol. 2019, 37, 527-530. [CrossRef] [PubMed] 
36. Harmsen, M.M.; Fijten, H.P.D.; Engel, B.; Dekker, A.; Eblé, P.L. Passive immunization with llama single-domain antibody fragments reduces foot-and-mouth disease transmission between pigs. Vaccine 2009, 27, 1904-1911. [CrossRef] [PubMed]

37. Virdi, V.; Coddens, A.; De Buck, S.; Millet, S.; Goddeeris, B.M.; Cox, E.; De Greve, H.; Depicker, A. Orally fed seeds producing designer igas protect weaned piglets against enterotoxigenic Escherichia coli infection. Proc. Natl. Acad. Sci. USA 2013, 110, 11809-11814. [CrossRef]

38. Harmsen, M.M.; van Solt, C.B.; Fijten, H.P.D.; van Keulen, L.; Rosalia, R.A.; Weerdmeester, K.; Cornelissen, A.H.M.; De Bruin, M.G.M.; Eblé, P.L.; Dekker, A. Passive immunization of guinea pigs with llama single-domain antibody fragments against foot-and-mouth disease. Vet. Microbiol. 2007, 120, 193-206. [CrossRef]

39. Schmidt, D.J.; Beamer, G.; Tremblay, J.M.; Steele, J.A.; Kim, H.B.; Wang, Y.; Debatis, M.; Sun, X.; Kashentseva, E.A.; Dmitriev, I.P.; et al. A tetraspecific VHH-based neutralizing antibody modifies disease outcome in three animal models of Clostridium difficile infection. Clin. Vaccine Immunol. 2016, 23, 774-784. [CrossRef]

40. Moonens, K.; De Kerpel, M.; Coddens, A.; Cox, E.; Pardon, E.; Remaut, H.; De Greve, H. Nanobody mediated inhibition of attachment of F18 fimbriae expressing Escherichia coli. PLoS ONE 2014, 9, e114691. [CrossRef]

41. Moonens, K.; Van Den Broeck, I.; Okello, E.; Pardon, E.; De Kerpel, M.; Remaut, H.; De Greve, H. Structural insight in the inhibition of adherence of F4 fimbriae producing Enterotoxigenic Escherichia coli by llama single domain antibodies. Vet. Res. 2015, 46, 1-7. [CrossRef]

42. Leenhouts, K.; Buist, G.; Kok, J. Anchoring of proteins to Lactic acid bacteria. Antonie van Leeuwenhoek 1999, 76, 367. [CrossRef] [PubMed]

43. O'Sullivan, D.J.; Klaenhammer, T.R. High- and low-copy-number Lactococcus shuttle cloning vectors with features for clone screening. Gene 1993, 137, 227-231. [CrossRef]

44. Gasson, M.J. Plasmid complements of Streptococcus lactis NCDO 712 and other lactic streptococci after protoplast-induced curing. J. Bacteriol. 1983, 154, 1-9. [CrossRef]

45. Cohen, S.; Sweeney, H.M. Modulation of protein A formation in Staphylococcus aureus by genetic determinants for methicillin resistance. J. Bacteriol. 1979, 140, 1028-1035. [CrossRef] [PubMed]

46. Raleigh, E.A.; Trimarchi, R.; Revel, H. Genetic and physical mapping of the mcrA (rglA) and mcrB (rglB) loci of Escherichia coli K-12. Genetics 1989, 122, 279-296. [CrossRef]

47. Cox, E.; Houvenaghel, A. Comparison of the in vitro adhesion of K88, K99, F41 and P987 positive Escherichia coli to intestinal villi of 4- to 5-week-old pigs. Vet. Microbiol. 1993, 34, 7-18. [CrossRef]

48. Van Den Broeck, W.; Cox, E.; Goddeeris, B.M. Receptor-specific binding of purified F4 to isolated villi. Vet. Microbiol. 1999, 68, 255-263. [CrossRef]

49. Okello, E.; Moonens, K.; Erume, J.; De Greve, H. Enterotoxigenic Escherichia coli strains are highly prevalent in Ugandan piggeries but disease outbreaks are masked by antibiotic prophylaxis. Trop. Anim. Health Prod. 2014, 47, 117-122. [CrossRef]

50. McCracken, A.; Turner, M.S.; Giffard, P.; Hafner, L.M.; Timms, P. Analysis of promoter sequences from Lactobacillus and Lactococcus and their activity in several Lactobacillus species. Arch. Microbiol. 2000, 173, 383-389. [CrossRef]

51. Esko, J.D.; Doering, T.L.; Raetz, C.R. Eubacteria and Archaea. In Essentials of Glycobiology, 2nd ed.; Varki, A., Cummings, R.D., Esko, J.D., Freeze, H.H., Stanley, P., Bertozzi, C.R., Hart, G.W., Etzler, M.E., Eds.; Cold Spring Harbor Laboratory Press: New York, NY, USA, 2009; Chapter 20; ISBN 9780879697709.

52. Schneewind, O.; Fowler, A.; Faull, K.F. Structure of the cell wall anchor of surface proteins in Staphylococcus aureus. Science 1995, 268, 103-106. [CrossRef]

53. Pant, N.; Hultberg, A.; Zhao, Y.; Svensson, L.; Pan-Hammarström, Q.; Johansen, K.; Pouwels, P.H.; Ruggeri, F.M.; Hermans, P.; Frenken, L.; et al. Lactobacilli expressing variable domain of Llama Heavy-chain antibody fragments (Lactobodies) confer protection against Rotavirus-induced diarrhea. J. Infect. Dis. 2006, 194, 1580-1588. [CrossRef]

54. De Greve, H.; Wyns, L.; Bouckaert, J. Combining sites of bacterial fimbriae. Curr. Opin. Struct. Biol. 2007, 17, 506-512. [CrossRef]

55. Van Den Broeck, W.; Cox, E.; Goddeeris, B.M. Receptor-dependent immune responses in pigs after oral immunization with F4 fimbriae. Infect. Immun. 1999, 67, 520-526. [CrossRef] [PubMed]

56. Klein, A.; Friedrich, U.; Vogelsang, H.; Jahreis, G. Lactobacillus acidophilus 74-2 and Bifidobacterium animalis subsp lactis DGCC 420 modulate unspecific cellular immune response in healthy adults. Eur. J. Clin. Nutr. 2008, 62, 584-593. [CrossRef]

57. Konstantinov, S.R.; Awati, A.A.; Williams, B.A.; Miller, B.G.; Jones, P.; Stokes, C.R.; Akkermans, A.D.L.; Smidt, H.; de Vos, W.M. Post-natal development of the porcine microbiota composition and activities. Environ. Microbiol. 2006, 8, 1191-1199. [CrossRef]

58. Lallès, J.P.; Bosi, P.; Smidt, H.; Stokes, C.R. Weaning-A challenge to gut physiologists. Livest. Sci. 2007, 108, 82-93. [CrossRef]

59. Rasschaert, K.; Verdonck, F.; Goddeeris, B.M.; Duchateau, L.; Cox, E. Screening of pigs resistant to F4 enterotoxigenic Escherichia coli (ETEC) infection. Vet. Microbiol. 2007, 123, 249-253. [CrossRef] [PubMed]

60. Miller, J. Experiments in Molecular Genetics; Cold Spring Harbor Laboratory: New York, NY, USA, $1972 ;$ ISBN 9780879691066.

61. Salmon, M.A.; Van Melderen, L.; Bernard, P.; Couturier, M. The antidote and autoregulatory functions of the F plasmid CcdA protein: A genetic and biochemical survey. MGG Mol. Gen. Genet. 1994, 244, 530-538. [CrossRef] [PubMed]

62. Gibson, D.G.; Young, L.; Chuang, R.Y.; Venter, J.C.; Hutchison, C.A.; Smith, H.O. Enzymatic assembly of DNA molecules up to several hundred kilobases. Nat. Methods 2009, 6, 343-345. [CrossRef] 